JOURNAL OF INTEGRAL EQUATIONS

AND APPLICATIONS

Volume 8, Number 2, Spring 1996

\title{
THE hp-VERSION OF THE BOUNDARY ELEMENT METHOD ON POLYGONS
}

\author{
N. HEUER AND E.P. STEPHAN
}

\begin{abstract}
We give a new proof for the exponential convergence of the hp-version of the boundary element method for some first kind integral equations on polygons. Crucial for our analysis are asymptotic expansions of the solutions of the integral equations in terms of singularity functions near the vertices of the polygon. The boundary integral equations under consideration are Symm's integral equation with the logarithmic kernel and the hypersingular integral equation resulting from taking the normal derivative of the double layer potential. Applications to acoustic scattering problems and crack problems in linear elasticity are given.
\end{abstract}

1. Introduction. The paper gives a further contribution to the analysis of the hp-version of the boundary element method (BEM) by presenting a more general result for Dirichlet and Neumann problems than [1] allowing the use of a general geometric mesh refinement on the polygonal boundary $\Gamma$. We give a new proof for the exponential convergence of the hp-version by exploiting only features of the solutions of the boundary integral equations. The key results in this approach are asymptotic expansions of the solutions of the integral equations in singularity functions reflecting the singular behavior of the solutions near corners of $\Gamma$. With the help of such expansions we show that the solutions of the integral equations belong to countably normed spaces. Therefore, these solutions can be approximated exponentially fast in the energy norm via the hp Galerkin solutions of those integral equations. This result is not restricted to integral equations which stem from boundary value problems for the Laplacian but applies to Helmholtz problems as well. Further applications are 2D crack problems in linear elasticity.

The paper is organized as follows. In Section 2 we recall the integral equations for the Dirichlet and the Neumann boundary value problem

Received by the editors on February 13, 1995, and in revised form on November 7, 1995.

AMS Subject Classifications. 45B05, 45E05, 45L10, 65R20.

Key words. hp-version of BEM, geometric mesh, exponential rate of convergence.

Copyright (C)1996 Rocky Mountain Mathematics Consortium 
of the Laplacian in a polygonal domain and introduce the Galerkin schemes. The behavior of the solutions of the integral equations near the corners of the polygon is studied in Section 3. In Section 4 we introduce the hp-version of the BEM and prove exponentially fast convergence for the Galerkin solution. In Section 5 we deal with a transmission problem from acoustic scattering of time harmonic waves. Section 6 presents some numerical experiments which confirm the theoretical results. Throughout the paper $c$ denotes a generic constant which may take different values at different occurrences.

2. Boundary integral equations. We consider boundary integral equation methods for solving Dirichlet and Neumann boundary value problems for the Laplacian in a polygonal domain $\Omega$ with boundary $\Gamma$. Let us assume that $\Gamma$ has conformal radius less than one; this can always be achieved by an appropriate scaling. Then the problems under consideration are the following ones:

Dirichlet problem. For given $f \in H^{1 / 2}(\Gamma)$ find $u \in H^{1}(\Omega)$ such that

$$
\Delta u=0 \quad \text { in } \Omega, \quad u=f \quad \text { on } \Gamma .
$$

Neumann problem. For given $g \in H^{-1 / 2}(\Gamma)$ with $\int_{\Gamma} g d s=0$, find $u \in H^{1}(\Omega)$ such that

$$
\Delta u=0 \quad \text { in } \Omega, \quad \partial u / \partial n=g \quad \text { on } \Gamma .
$$

Here $\partial u / \partial n$ denotes the normal derivative of $u$ with respect to the outer normal $n$. It is well-known that problems (1) and (2) can be converted into boundary integral equations of the first kind on $\Gamma$, cf. [2]. With $v=\left.u\right|_{\Gamma}, \psi=\partial u /\left.\partial n\right|_{\Gamma}$, we have for (1) and (2), respectively,

$$
\begin{array}{ll}
V \psi=(1+K) f & \text { on } \Gamma \\
D v=\left(1-K^{\prime}\right) g & \text { on } \Gamma
\end{array}
$$


with the integral operators (for $\phi \in H^{-1 / 2}(\Gamma), w \in H^{1 / 2}(\Gamma)$ )

$$
\begin{aligned}
V \phi(x) & :=-\frac{1}{\pi} \int_{\Gamma} \phi(y) \log |x-y| d s_{y}, \\
K w(x) & :=-\frac{1}{\pi} \int_{\Gamma} w(y) \frac{\partial}{\partial n_{y}} \log |x-y| d s_{y}, \\
K^{\prime} \phi(x) & :=-\frac{1}{\pi} \int_{\Gamma} \phi(y) \frac{\partial}{\partial n_{x}} \log |x-y| d s_{y}, \\
D w(x) & :=\frac{1}{\pi} \frac{\partial}{\partial n_{x}} \int_{\Gamma} w(y) \frac{\partial}{\partial n_{y}} \log |x-y| d s_{y} .
\end{aligned}
$$

It is also well-known that there exist unique solutions $\psi \in H^{-1 / 2}(\Gamma)$ of (3) and

$$
v \in H_{0}^{1 / 2}(\Gamma)=\left\{w \in H^{1 / 2}(\Gamma) ; \int_{\Gamma} w d s=0\right\}
$$

of (4). The boundary integral operators $V$ and $D$ are strongly elliptic pseudodifferential operators satisfying a Gårding's inequality on $H^{-1 / 2}(\Gamma)$ and $H_{0}^{1 / 2}(\Gamma)$, respectively. Therefore, due to [12], any conforming Galerkin scheme for (3) and (4) converges quasioptimally in the energy norm. Let $X_{N}$ and $Y_{N}$ denote subspaces of dimension $N$ of $X:=H^{-1 / 2}(\Gamma)$ and $Y:=H_{0}^{1 / 2}(\Gamma)$. Then the Galerkin schemes read:

Find $\psi_{N} \in X_{N}$ satisfying

$$
\left\langle V \psi_{N}, \phi\right\rangle_{L^{2}(\Gamma)}=\langle(1+K) f, \phi\rangle_{L^{2}(\Gamma)}, \quad \forall \phi \in X_{N},
$$

find $v_{N} \in Y_{N}$ satisfying

$$
\left\langle D v_{N}, w\right\rangle_{L^{2}(\Gamma)}=\left\langle\left(I-K^{\prime}\right) g, w\right\rangle_{L^{2}(\Gamma)}, \quad \forall w \in Y_{N} .
$$

Then for the Galerkin solutions $\psi_{N}, v_{N}$ and the true solutions $\psi$ and $v$, there holds

$$
\left\|\psi-\psi_{N}\right\|_{H^{-1 / 2}(\Gamma)} \leq c_{1}\|\psi-\phi\|_{H^{-1 / 2}(\Gamma)}, \quad \forall \phi \in X_{N}
$$

and

$$
\left\|v-v_{N}\right\|_{H^{1 / 2}(\Gamma)} \leq c_{2}|| v-w \|_{H^{1 / 2}(\Gamma)}, \quad \forall w \in Y_{N}
$$


where the constants $c_{1}, c_{2}>0$ are independent of $N$.

It is shown in [11] that the h-version and the p-version of (5) and (6) on a quasiuniform mesh have only algebraic rates of convergence, whereas it is shown in [1] that the hp-version on geometric meshes converges exponentially fast. However in $[\mathbf{1}]$ the boundary element mesh is the trace on $\Gamma$ of a geometric mesh in $\Omega$ and the boundary elements on $\Gamma$ must be the traces or normal derivatives on $\Gamma$ of finite element functions in $\Omega$. This means a restriction on the choice of boundary elements and on the construction of the geometric mesh refinement on the boundary $\Gamma$. Here we give a new proof of the exponential convergence of the hp-version of the boundary element method which does not require these restrictions. The analysis given here can be extended, e.g., to curved polygons $\Gamma$ and to the Helmholtz operator in (1) and (2) (instead of the Laplacian) as shown below.

3. Regularity of the solutions of the integral equations. In this section we prove special expansions of the solutions of the integral equations. These expansions will be used in Section 4 to prove the exponentially fast convergence of the hp-version of the BEM with geometric meshes. The main result of this section is the following theorem which uses the method of Mellin transformation as presented in $[3]$.

Theorem 1. Provided $f$ is piecewise analytic the solution $\psi$ of (3) has the form

$$
\begin{aligned}
& \psi(x)=\sum_{j=1}^{J} \sum_{k=1}^{n}\left(c_{k}^{j, 1}\left|x-x_{j}\right|^{\alpha_{k_{j}}-1}\right. \\
& \left.+c_{k}^{j, 2}\left|x-x_{j}\right|^{\alpha_{k_{j}}-1} \log \left|x-x_{j}\right|\right) \chi_{j}(x)+\psi_{0}(x), \\
& x \in \Gamma, \quad c_{k}^{j, 1}, c_{k}^{j, 2} \in \mathbf{R}^{2}, \quad \alpha_{k j}=k \frac{\pi}{w_{j}}, \quad n \leq \frac{\omega_{j}}{\pi}(t-3 / 2)
\end{aligned}
$$

where $\left.\psi_{0}\right|_{\Gamma^{j}} \in H^{t-1}\left(\Gamma^{j}\right)$ depends on $t$. For the coefficients $c_{k}^{j, 1}$ and $c_{k}^{j, 2}$ there hold the relations (40). The solution $v$ of (4) for piecewise 
analytic $g$ has the form

$$
\begin{aligned}
v(x)=\sum_{j=1}^{J} \sum_{k=1}^{n}\left(d_{k}^{j, 1}\left|x-x_{j}\right|^{\alpha_{k_{j}}}\right. & \\
+ & \left.d_{k}^{j, 2}\left|x-x_{j}\right|^{\alpha_{k_{j}}} \log \left|x-x_{j}\right|\right) \chi_{j}(x)+v_{0}(x), \\
x & \in \Gamma, \quad d_{k}^{j, 1}, d_{k}^{j, 2} \in \mathbf{R}^{2}
\end{aligned}
$$

with $\left.v_{0}\right|_{\Gamma^{j}} \in H^{t}\left(\Gamma^{j}\right)$ depending on $t$. For the coefficients $d_{k}^{j, 1}$ and $d_{k}^{j, 2}$ there hold the relations (41). Here $\chi_{j}$ is a $C^{\infty}$ cut-off function concentrated at the $j$ th corner $x_{j}$, with opening angle $\omega_{j}$. The twodimensional coefficients $c_{k}^{j}=\left(-c_{k}^{j},{ }^{+} c_{k}^{j}\right)^{T}$ and $d_{k}^{j}=\left({ }^{-} d_{k}^{j},{ }^{+} d_{k}^{j}\right)^{T}$ are to be understood in the sense that we have to take $c_{k}^{j}={ }^{-} c_{k}^{j}$ and $d_{k}^{j}={ }^{-} d_{k}^{j}$ on $\Gamma_{j-1}$ and $c_{k}^{j}={ }^{+} c_{k}^{j}$ and $d_{k}^{j}={ }^{+} d_{k}^{j}$ on $\Gamma_{j}$, where $\Gamma_{j-1}$ and $\Gamma_{j}$ are the edges of the polygon meeting at the corner $x_{j}$.

Before we prove the theorem we cite from [3] two lemmas which characterize the use of the Mellin transform.

Lemma 1 [3, Lemma 4.1]. Suppose that

$$
f(x)=\left(\sum_{k=1}^{n} \sum_{l=0}^{l_{k}} c_{k l} x^{\alpha_{k}} \log ^{l} x\right) \chi(x)+f_{0}(x), \quad x \in \mathbf{R}_{+}
$$

where $f_{0} \in C_{0}^{\infty}(0, \infty), \chi \in C_{0}^{\infty}[0, \infty)$ with $\operatorname{supp}(1-\chi) \subset(0, \infty)$, $\alpha_{1}<\alpha_{2}<\cdots<\alpha_{n}$. Then

(i) The Mellin transform

$$
\hat{f}(\lambda):=\int_{0}^{\infty} x^{i \lambda-1} f(x) d x
$$

exists and is analytic for $\Im(\lambda)<\alpha_{1}$, and it has a meromorphic extension on $\mathbf{C}$ with poles at $\lambda=i \alpha_{k}, k=1, \ldots, n$, of order $l_{k}+1$.

(ii) In the strip $\left\{\lambda \in \mathbf{C} ; \Im(\lambda) \in\left(\alpha_{1}, \alpha_{j+1}\right)\right\}, \hat{f}$ is the Mellin transform of $f_{j}$ defined by

$$
f_{j}(x)=f(x)-\sum_{k=1}^{j} \sum_{l=0}^{l_{k}} c_{k l} x^{\alpha_{k}} \log ^{l} x .
$$


(iii) If we define

$$
\gamma_{r}^{\lambda_{0}}(\hat{f}):=\operatorname{Res}_{\lambda=\lambda_{0}} \frac{\hat{f}(\lambda)}{\left(\lambda-\lambda_{0}\right)^{r}}, \quad r \in \mathbf{Z},
$$

then we have

$$
\begin{aligned}
\gamma_{r}^{\lambda_{0}}(\hat{f}) & =\left.\frac{1}{\left(l_{k}+r\right) !}\left(\frac{d}{d \lambda}\right)^{l_{k}+r}\left[\hat{f}(\lambda)\left(\lambda-\lambda_{0}\right)^{l_{k}+1}\right]\right|_{\lambda=\lambda_{0}} \\
& =\frac{1}{2 \pi i}\left(\int_{\Im(\lambda)=h_{1}}-\int_{\Im(\lambda)=h_{2}}\right) \frac{\hat{f}(\lambda)}{\left(\lambda-\lambda_{0}\right)^{r}} d \lambda,
\end{aligned}
$$

where $\lambda_{0}=i \alpha_{k}, l_{k}+r \geq 0, \alpha_{k-1}<h_{1}<\alpha_{k}<h_{2}<\alpha_{k+1} ; \gamma_{r}^{\lambda_{0}}(\hat{f})=0$ if $l_{k}+r<0$ or if $\hat{f}$ is regular at $\lambda_{0}$;

$$
\gamma_{-l}^{i \alpha_{k}}(\hat{f})=-i^{l+1} l ! c_{k l}, \quad 0 \leq l \leq l_{k}, k=1, \ldots, n .
$$

Lemma 2 [3, Lemma 4.3]. Let $\hat{f}$ be meromorphic in a strip $\Im(\lambda) \in$ $\left(\alpha_{0}, \alpha_{n+1}\right)$ and have poles at $\lambda=i \alpha_{k}$ of order $l_{k}+1, k=1, \ldots, n$, $\alpha_{0}<\alpha_{1}<\cdots<\alpha_{n+1}$. Assume that, for $\Im(\lambda)=$ const, $\hat{f}(\lambda)$ is rapidly decreasing as $|\Re(\lambda)| \rightarrow \infty$. Define $f_{(h)}$ by the inverse Mellin transform

$$
f_{(h)}(x):=\frac{1}{2 \pi} \int_{\Im(\lambda)=h} e^{i \lambda t} \hat{f}(\lambda) d \lambda, \quad x=e^{-t} \in \mathbf{R}_{+} .
$$

Then for $h \in\left(\alpha_{0}, \alpha_{1}\right)$ and $h^{\prime} \in\left(\alpha_{n}, \alpha_{n+1}\right)$, we have

$$
\begin{aligned}
f_{(h)} & \in \tilde{H}^{s}\left(\mathbf{R}_{+}\right) \quad \text { for } s-1 / 2 \in\left(\alpha_{0}, \alpha_{1}\right) ; \\
f_{\left(h^{\prime}\right)} & \in \tilde{H}^{s^{\prime}}\left(\mathbf{R}_{+}\right) \quad \text { for } s^{\prime}-1 / 2 \in\left(\alpha_{n}, \alpha_{n+1}\right)
\end{aligned}
$$

and

$$
f_{(h)}(x)=\sum_{k=1}^{n} \sum_{l=0}^{l_{k}} c_{k l} x^{\alpha_{k}} \log ^{l} x+f_{\left(h^{\prime}\right)}(x) .
$$

Formula (11) holds in this case also. 
Remark 1. In $[\mathbf{3}]$ there were spaces $\stackrel{\circ}{W}_{0}^{s}$ considered for $(12)$ and (13) which are different from the Sobolev spaces $\tilde{H}^{s}\left(\mathbf{R}_{+}\right)$but have equivalent norms on compact sets. Since we are dealing with functions with compact supports, this formulation is appropriate.

Proof of Theorem 1. Following Costabel and Stephan [4] we use the method of Mellin transformation to expand the solutions of the integral equations (3) and (4) near the corners of the polygon. Let us consider the reference angle $\Gamma^{\omega}=\Gamma^{-} \cup\{0\} \cup \Gamma^{+}$with $\Gamma^{-}=e^{i \omega} \mathbf{R}_{+}$and $\Gamma^{+}=\mathbf{R}_{+}$, $\omega \in(0,2 \pi)$. A function $\phi$ on $\Gamma^{\omega}$ can be identified with the pair $\left(\phi_{-}, \phi_{+}\right)$ of functions on $\mathbf{R}_{+}$defined by $\phi_{-}(x)=\phi\left(x e^{i \omega}\right), \phi_{+}(x)=\phi(x), x>0$. We will choose the representation of $\phi$ by its even and odd parts which are defined by

$$
\phi^{e}=\frac{1}{2}\left(\phi_{-}+\phi_{+}\right), \quad \phi^{o}=\frac{1}{2}\left(\phi_{-}-\phi_{+}\right) .
$$

This induces for any operator $A$ acting on functions on $\Gamma^{\omega}$ a representation by a $2 \times 2$ matrix of operators acting on functions on $\mathbf{R}_{+}$:

$$
A \hat{=} \mathcal{A}:=\left(\begin{array}{cc}
A_{e e} & A_{o e} \\
A_{\text {eo }} & A_{o o}
\end{array}\right)
$$

where

$$
\begin{aligned}
& (A \phi)^{e}=A_{e e} \phi^{e}+A_{o e} \phi^{o}, \\
& (A \phi)^{o}=A_{e o} \phi^{e}+A_{o o} \phi^{o} .
\end{aligned}
$$

We need the following operators acting on functions on $\mathbf{R}_{+}$:

$$
\begin{aligned}
V_{\omega} \phi(x):= & -\frac{1}{\pi} \int_{0}^{\infty} \log \left|1-\frac{x}{y} e^{-i \omega}\right| \phi(y) d y, \\
& V_{0}=V_{\omega} \text { for } \omega=0 . \\
K_{\omega} \phi(x):= & \frac{1}{\pi} \int_{0}^{\infty} \Im\left(\frac{1}{x e^{i \omega}-y}\right) \phi(y) d y, \\
K_{\omega}^{\prime} \phi(x):= & \frac{1}{\pi} \int_{0}^{\infty} \Im\left(\frac{e^{i \omega}}{x e^{i \omega}-y}\right) \phi(y) d y, \\
D_{\omega} \phi(x):= & -\frac{1}{x} \frac{\partial}{\partial \omega} K_{\omega} \phi(x), \quad D_{0}=\lim _{\omega \rightarrow 0} D_{\omega} .
\end{aligned}
$$


Then, with the exception of finite dimensional operators which are neglectible in our theory, the integral operators $V, D, K$ and $K^{\prime}$ can be represented by the following matrices (see [4]):

$$
\begin{aligned}
V \hat{=} \mathcal{V} & =\left(\begin{array}{cc}
V_{0}+V_{\omega} & 0 \\
0 & V_{0}-V_{\omega}
\end{array}\right) \\
D \hat{=} \mathcal{D} & =\left(\begin{array}{cc}
D_{\omega}-D_{0} & 0 \\
0 & -\left(D_{0}+D_{\omega}\right)
\end{array}\right) \\
K \hat{=} \mathcal{K} & =\left(\begin{array}{cc}
K_{\omega} & 0 \\
0 & -K_{\omega}
\end{array}\right) \\
K^{\prime} \hat{=} \mathcal{K}^{\prime} & =\left(\begin{array}{cc}
K_{\omega}^{\prime} & 0 \\
0 & -K_{\omega}^{\prime}
\end{array}\right)
\end{aligned}
$$

For these representations the Mellin symbols are known explicitly (see $[4])$ :

$$
\begin{aligned}
\mathcal{M}\left(V_{\omega} \phi\right)(\lambda) & =\hat{V}_{\omega}(\lambda) \hat{\phi}(\lambda-i) \\
& :=\frac{\cosh [(\pi-\omega) \lambda]}{\lambda \sinh \pi \lambda} \hat{\phi}(\lambda-i), \quad \Im(\lambda) \in(0,1),
\end{aligned}
$$

$$
\begin{aligned}
& \mathcal{M}\left(D_{\omega} \phi\right)(\lambda)=\hat{D}_{\omega}(\lambda+i) \hat{\phi}(\lambda+i) \\
& :=-(\lambda+i) \frac{\cosh [(\pi-\omega)(\lambda+i)]}{\sinh [\pi(\lambda+i)]} \hat{\phi}(\lambda+i), \quad \Im(\lambda) \in(-2,0), \\
& \mathcal{M}\left(K_{\omega} \phi\right)(\lambda)=\hat{K}_{\omega}(\lambda) \hat{\phi}(\lambda) \\
& :=-\frac{\sinh [(\pi-\omega) \lambda]}{\sinh \pi \lambda} \hat{\phi}(\lambda), \quad \Im(\lambda) \in(-1,1), \\
& (20) \quad \mathcal{M}\left(K_{\omega}^{\prime} \phi\right)(\lambda)=\hat{K}_{\omega}(\lambda+i) \hat{\phi}(\lambda), \quad \Im(\lambda) \in(-2,0) .
\end{aligned}
$$

First let us consider the weakly singular integral equation (3). Acting on even and odd functions on $\Gamma_{\omega}$, this equation becomes

$$
\left(\begin{array}{cc}
V_{0}+V_{\omega} & 0 \\
0 & V_{0}-V_{\omega}
\end{array}\right)\left(\begin{array}{l}
\psi^{e} \\
\psi^{o}
\end{array}\right)=\left(\begin{array}{cc}
1+K_{\omega} & 0 \\
0 & 1-K_{\omega}
\end{array}\right)\left(\begin{array}{l}
f^{e} \\
f^{o}
\end{array}\right)
$$


Applying the Mellin transformation, we obtain

$$
\begin{aligned}
& \left(\begin{array}{c}
\hat{\psi}^{e}(\lambda-i) \\
\hat{\psi}^{o}(\lambda-i)
\end{array}\right) \\
& =\left(\begin{array}{cc}
\left(\frac{\cosh \pi \lambda+\cosh [(\pi-\omega) \lambda]}{\lambda \sinh \pi \lambda}\right) & 0 \\
0 & \left(\frac{\cosh \pi \lambda-\cosh [(\pi-\omega) \lambda]}{\lambda \sinh \pi \lambda}\right)
\end{array}\right)^{-1} \\
& \times\left(\begin{array}{cc}
1-\frac{\sinh [(\pi-\omega) \lambda]}{\sinh \pi \lambda} & 0 \\
0 & 1+\frac{\sinh [(\pi-\omega) \lambda]}{\sinh \pi \lambda}
\end{array}\right)\left(\begin{array}{c}
\hat{f}^{e}(\lambda) \\
\hat{f}^{o}(\lambda)
\end{array}\right) \\
& =\lambda\left(\begin{array}{cc}
\frac{\sinh \pi \lambda-\sinh [(\pi-\omega) \lambda]}{\cosh \pi \lambda+\cosh [(\pi-\omega) \lambda]} & 0 \\
0 & \frac{\sinh \pi \lambda+\sinh [(\pi-\omega) \lambda]}{\cosh \pi \lambda-\cosh [(\pi-\omega) \lambda]}
\end{array}\right) \\
& \times\left(\begin{array}{l}
\hat{f}^{e}(\lambda) \\
\hat{f}^{o}(\lambda)
\end{array}\right) \\
& =\lambda\left(\begin{array}{cc}
\frac{\sinh (\omega / 2) \lambda \cosh [(\pi-\omega / 2) \lambda]}{\cosh [(\pi-\omega / 2) \lambda] \cosh (\omega / 2) \lambda} & 0 \\
0 & \frac{\sinh [(\pi-\omega / 2) \lambda] \cosh (\omega / 2) \lambda}{\sinh [(\pi-\omega / 2) \lambda] \sinh (\omega / 2) \lambda}
\end{array}\right) \\
& \times\left(\begin{array}{l}
\hat{f}^{e}(\lambda) \\
\hat{f}^{o}(\lambda)
\end{array}\right) \\
& =\lambda\left(\begin{array}{cc}
\frac{\sinh (\omega / 2) \lambda}{\cosh (\omega / 2) \lambda} & 0 \\
0 & \frac{\cosh (\omega / 2) \lambda}{\sinh (\omega / 2) \lambda}
\end{array}\right)\left(\begin{array}{l}
\hat{f}^{e}(\lambda) \\
\hat{f}^{o}(\lambda)
\end{array}\right)
\end{aligned}
$$

Due to the piecewise analyticity of $f$ near the corners, the even and odd parts of $f$ have expansions of the form

$$
f^{e}(x)=\sum_{k=0}^{\infty} f_{k}^{e} x^{k}, \quad f^{o}(x)=\sum_{k=1}^{\infty} f_{k}^{o} x^{k} .
$$

Note that $f^{o}=(1 / 2)\left(f_{-}-f_{+}\right)$has no constant term since $f$ is continuous at the corners. Therefore, $\hat{f}^{e}$ has first order poles at $i k$, 
$k=0,1, \ldots$ and $\hat{f}^{o}$ has first order poles at $i k, k=1,2, \ldots$ (cf. Lemma 1). The zeros of $\cosh (\omega / 2) \lambda$ and $\sinh (\omega / 2) \lambda$ are $i(2 k+1)(\pi / \omega)$ and $i 2 k(\pi / \omega)$ ( $k$ integer $)$, respectively. Now

$$
\hat{\psi}^{e}(\lambda)=(\lambda+i) \frac{\sinh [(\omega / 2)(\lambda+i)]}{\cosh [(\omega / 2)(\lambda+i)]} \hat{f}^{e}(\lambda+i)
$$

has poles at

$$
i((2 k+1)(\pi / \omega)-1) \quad \text { and at } i k, \quad k=0,1, \ldots
$$

There is no pole at $-i \operatorname{since} \sinh [(\omega / 2)(\lambda+i)]$ and $(\lambda+i)$ are zero there. Note further that the poles at $\lambda$ with $\Im(\lambda) \leq-1$ are not taken into account since we already know that $\psi \in H^{-1 / 2}+\varepsilon(\Gamma), \varepsilon>0$, due to the piecewise analyticity of $f, \mathrm{cf}$. [2]. Some of the poles are of second order if $\pi / \omega$ is rational.

Analogously,

$$
\hat{\psi}^{o}(\lambda)=(\lambda+i) \frac{\cosh [(\omega / 2)(\lambda+i)]}{\sinh [(\omega / 2)(\lambda+i)]} \hat{f}^{o}(\lambda+i)
$$

has poles at

$$
\begin{gathered}
i(2 k(\pi / \omega)-1), \quad k=1,2, \ldots \\
\text { and at } i k, \quad k=0,1, \ldots .
\end{gathered}
$$

Thus, we obtain for $\psi^{e}$ and $\psi^{o}$ the expansions (cf. Lemma 2)

$$
\begin{aligned}
\psi^{e}(x)= & \sum_{k=0}^{n}\left(\psi_{k}^{e, 1} x^{(2 k+1)(\pi / \omega)-1}+\psi_{k}^{e, 2} x^{(2 k+1)(\pi / \omega)-1} \log |x|\right) \\
& +\sum_{0 \leq k<(2 n+1)(\pi / \omega)-1} \psi_{k}^{e, 3} x^{k}+\psi_{0}^{e}(x)
\end{aligned}
$$

and

$$
\begin{aligned}
\psi^{o}(x)= & \sum_{k=1}^{n}\left(\psi_{k}^{o, 1} x^{2 k(\pi / \omega)-1}+\psi_{k}^{o, 2} x^{2 k(\pi / \omega)-1} \log |x|\right) \\
& +\sum_{0 \leq k<2 n(\pi / \omega)-1} \psi_{k}^{o, 3} x^{k}+\psi_{0}^{o}(x)
\end{aligned}
$$


where

$$
\psi_{0}^{e} \in \tilde{H}^{s}\left(\mathbf{R}_{+}\right) \quad \text { for } s-1 / 2 \in((2 n+1)(\pi / \omega)-1,[(2 n+1)(\pi / \omega)])
$$

and

$$
\psi_{0}^{o} \in \tilde{H}^{s}\left(\mathbf{R}_{+}\right) \quad \text { for } s-1 / 2 \in(2 n(\pi / \omega)-1,[2 n(\pi / \omega)]) .
$$

Here $[m]$ denotes the largest integer less than or equal to $m$. Of course, the sums just before the remainders $\psi_{0}^{e}$ and $\psi_{0}^{o}$ are polynomials and therefore arbitrarily smooth. However, since we are interested in the expansion of $\psi^{e}$ and $\psi^{o}$ for $n \rightarrow \infty$ we have to take care of these additional terms. It remains to estimate the coefficients $\psi_{k}^{e, 1}, \psi_{k}^{e, 2}, \psi_{k}^{e, 3}$ and $\psi_{k}^{o, 1}, \psi_{k}^{o, 2}, \psi_{k}^{o, 3}$. First of all we note that the coefficients of the polynomials corresponding to second order poles are zero. This is so because, in this case, they are already taken into account by the respective terms in the first series. Further, in the case of first order poles the respective coefficients $\psi_{k}^{e, 2}$ and $\psi_{k}^{o, 2}$ are zero. Let us concentrate on $\psi^{e}$. The coefficients of the expansion of $\psi^{o}$ can be estimated analogously.

Applying relation (11), we conclude

$$
\begin{aligned}
\left|\psi_{k}^{e, 1}\right| & =\left|\underset{\lambda=i((2 k+1)(\pi / \omega)-1)}{\operatorname{Res}} \hat{\psi}^{e}(\lambda)\right| \\
& =\left|\underset{\lambda=i(2 k+1)(\pi / \omega)}{\operatorname{Res}} \hat{\psi}^{e}(\lambda-i)\right| \\
& =\left|\operatorname{Res}_{\lambda=i(2 k+1)(\pi / \omega)} \lambda \frac{\sinh (\omega / 2) \lambda}{\cosh (\omega / 2) \lambda} \hat{f}^{e}(\lambda)\right| .
\end{aligned}
$$

Now, if $i(2 k+1)(\pi / \omega)$ is no pole of $\hat{f}^{e}$, i.e., $(2 k+1)(\pi / \omega)$ is not an integer,

$$
\left|\underset{\lambda=i(2 k+1)(\pi / \omega)}{\operatorname{Res}} \lambda \frac{\sinh (\omega / 2) \lambda}{\cosh (\omega / 2) \lambda} \hat{f}^{e}(\lambda)\right|=(2 k+1) \frac{\pi}{\omega} \frac{2}{\omega}\left|\hat{f}^{e}\left(i(2 k+1) \frac{\pi}{\omega}\right)\right|
$$

and in the case $i(2 k+1)(\pi / \omega)$ is a pole of $\hat{f}^{e}$ we obtain

$$
\begin{aligned}
& \left|\underset{\lambda=i(2 k+1)(\pi / \omega)}{\operatorname{Res}} \lambda \frac{\sinh (\omega / 2) \lambda}{\cosh (\omega / 2) \lambda} \hat{f}^{e}(\lambda)\right| \\
& =\left|\operatorname{Res}_{\lambda=i(2 k+1)(\pi / \omega)} \lambda \frac{\sinh (\omega / 2) \lambda}{\cosh (\omega / 2) \lambda}\left(\hat{f}^{e}(\lambda)-\frac{\operatorname{Res}_{\lambda=i(2 k+1)(\pi / \omega)} \hat{f}^{e}(\lambda)}{\lambda-i(2 k+1)(\pi / \omega)}\right)\right|
\end{aligned}
$$


since

$$
\operatorname{Res}_{\lambda=i(2 k+1)(\pi / \omega)}\left(\lambda \frac{\sinh (\omega / 2) \lambda}{\cosh (\omega / 2) \lambda} \frac{1}{\lambda-i(2 k+1)(\pi / \omega)}\right)=0 .
$$

Therefore, in the latter case,

$$
\begin{aligned}
\left|\psi_{k}^{e, 1}\right| & =(2 k+1) \frac{2 \pi}{\omega^{2}}\left|\hat{f}^{e}(\lambda)-\frac{\operatorname{Res}_{\lambda=i(2 k+1)(\pi / \omega)} \hat{f}^{e}(\lambda)}{\lambda-i(2 k+1)(\pi / \omega)}\right|_{\lambda=i(2 k+1)(\pi / \omega)} \\
& =(2 k+1) \frac{2 \pi}{\omega^{2}}\left|\hat{f}^{e}(\lambda)-\frac{f_{(2 k+1)(\pi / \omega)}^{e}}{\lambda-i(2 k+1)(\pi / \omega)}\right|_{\lambda=i(2 k+1)(\pi / \omega)} .
\end{aligned}
$$

Provided $i(2 k+1)(\pi / \omega)$ is a pole of $\hat{f}^{e}$ we obtain with the help of formula (11)

$$
\begin{aligned}
& \left|\psi_{k}^{e, 2}\right|=\left|\operatorname{Res}_{\lambda=i(2 k+1)(\pi / \omega)}\left(\lambda-i(2 k+1) \frac{\pi}{\omega}\right) \hat{\psi}^{e}(\lambda-i)\right| \\
& =\left|\operatorname{Res}_{\lambda=i(2 k+1)(\pi / \omega)}\left(\lambda-i(2 k+1) \frac{\pi}{\omega}\right) \lambda \frac{\sinh (\omega / 2) \lambda}{\cosh (\omega / 2) \lambda} \hat{f}^{e}(\lambda)\right| \\
& =\lim _{y \rightarrow(2 k+1)(\pi / \omega)}\left|\left(y-(2 k+1) \frac{\pi}{\omega}\right)^{2} y \frac{\sin (\omega / 2) y}{\cos (\omega / 2) y} \hat{f}^{e}(i y)\right| \\
& =\lim _{y \rightarrow(2 k+1)(\pi / \omega)} \mid\left(y-(2 k+1) \frac{\pi}{\omega}\right)^{2} y \frac{\sin (\omega / 2) y}{\cos (\omega / 2) y} \\
& \frac{\operatorname{Res}_{\lambda=i(2 k+1)(\pi / \omega)} \hat{f}^{e}(\lambda)}{y-(2 k+1)(\pi / \omega)} \mid
\end{aligned}
$$

since

$$
\begin{aligned}
\operatorname{Res}_{\lambda=i(2 k+1)(\pi / \omega)}\left[(\lambda-i(2 k+1)(\pi / \omega)) \lambda \frac{\sinh (\omega / 2) \lambda}{\cosh (\omega / 2) \lambda}\right. \\
\left.\left(\hat{f}^{e}(\lambda)-\frac{\operatorname{Res}_{\lambda=i(2 k+1)(\pi / \omega)} \hat{f}^{e}(\lambda)}{\lambda-i(2 k+1)(\pi / \omega)}\right)\right]=0 .
\end{aligned}
$$

Therefore,

$$
\begin{aligned}
\left|\psi_{k}^{e, 2}\right| & =\lim _{y \rightarrow(2 k+1)(\pi / \omega)}\left|\left(y-(2 k+1) \frac{\pi}{\omega}\right) y \frac{\sin (\omega / 2) y}{\cos (\omega / 2) y} \operatorname{Res}_{i(2 k+1)(\pi / \omega)} \hat{f}^{e}(\lambda)\right| \\
& =(2 k+1) \frac{2 \pi}{\omega^{2}}\left|f_{(2 k+1)(\pi / \omega)}^{e}\right| .
\end{aligned}
$$


For the coefficient $\psi_{k}^{e, 3}$ there holds, if $i(k+1)$ is no pole of $1 / \cosh (\omega / 2) \lambda$,

$$
\begin{aligned}
\left|\psi_{k}^{e, 3}\right| & =\left|\operatorname{Res}_{\lambda=i k} \hat{\psi}^{e}(\lambda)\right|=\left|\operatorname{Res}_{\lambda=i(k+1)} \hat{\psi}^{e}(\lambda-i)\right| \\
& =\left|\operatorname{Res}_{\lambda=i(k+1)} \lambda \frac{\sinh (\omega / 2) \lambda}{\cosh (\omega / 2) \lambda} \hat{f}^{e}(\lambda)\right| \\
& =(k+1)\left|\frac{\sin (\omega / 2)(k+1)}{\cos (\omega / 2)(k+1)}\right|\left|f_{k+1}^{e}\right| .
\end{aligned}
$$

Summing up, we proved

(26)

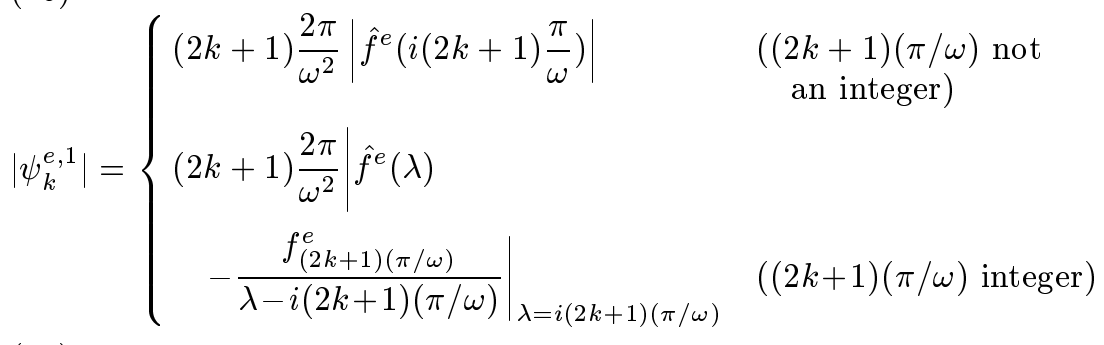

$$
\left|\psi_{k}^{e, 2}\right|= \begin{cases}0 & ((2 k+1)(\pi / \omega) \text { not an integer }) \\ (2 k+1)\left(2 \pi / \omega^{2}\right)\left|f_{(2 k+1) \pi / \omega}^{e}\right| & ((2 k+1)(\pi / \omega) \text { integer })\end{cases}
$$

$$
\left|\psi_{k}^{e, 3}\right|=\left\{\begin{array}{ll}
(k+1)\left|\frac{\sin (\omega / 2)(k+1)}{\cos (\omega / 2)(k+1)}\right|\left|f_{k+1}^{e}\right| & (k+1 \neq(2 m+1)(\pi / \omega) \\
& \text { for all integers } m) \\
0 & \text { (else) }
\end{array} .\right.
$$

Similarly, we find for the coefficients of $\psi^{o}$

$$
\left|\psi_{k}^{o, 1}\right|=\left\{\begin{array}{lr}
2 k\left(2 \pi / \omega^{2}\right) \mid \hat{f}^{o}(i 2 k(\pi / \omega) \mid & (2 k(\pi / \omega) \text { not an integer }) \\
2 k\left(2 \pi / \omega^{2}\right) \mid \hat{f}^{o}(\lambda) & (2 k(\pi / \omega) \text { integer }) \\
-\left.\frac{f_{2 k(\pi / \omega)}^{o}}{\lambda-i 2 k(\pi / \omega)}\right|_{\lambda=i 2 k(\pi / \omega)} &
\end{array}\right.
$$


(30)

$$
\begin{aligned}
& \left|\psi_{k}^{o, 2}\right|= \begin{cases}0 & (2 k(\pi / \omega) \text { not an integer }) \\
2 k\left(2 \pi / \omega^{2}\right)\left|f_{2 k(\pi / \omega)}^{o}\right| & (2 k(\pi / \omega) \text { integer })\end{cases} \\
& \left|\psi_{k}^{o, 3}\right|= \begin{cases}(k+1)\left|\frac{\cos (\omega / 2)(k+1)}{\sin (\omega / 2)(k+1)}\right|\left|f_{k+1}^{o}\right| & (k+1 \neq 2 m(\pi / \omega) \\
& \text { for all integers } m) . \\
0 & \text { (else) }\end{cases}
\end{aligned}
$$

Analogously, the hypersingular integral equation (4) becomes

$$
\left(\begin{array}{cc}
D_{\omega}-D_{0} & 0 \\
0 & -\left(D_{0}+D_{\omega}\right)
\end{array}\right)\left(\begin{array}{l}
v^{e} \\
v^{o}
\end{array}\right)=\left(\begin{array}{cc}
1-K_{\omega}^{\prime} & 0 \\
0 & 1+K_{\omega}^{\prime}
\end{array}\right)\left(\begin{array}{l}
g^{e} \\
g^{o}
\end{array}\right)
$$

and using the Mellin transformation, we obtain

$$
\begin{aligned}
& \left(\begin{array}{l}
\hat{v}^{e}(\lambda) \\
\hat{v}^{o}(\lambda)
\end{array}\right) \\
& =\left(\begin{array}{cc}
\lambda\left(\frac{\cosh \pi \lambda-\cosh [(\pi-\omega) \lambda]}{\sinh \pi \lambda}\right) & 0 \\
0 & \lambda\left(\frac{\cosh \pi \lambda+\cosh [(\pi-\omega) \lambda]}{\sinh \pi \lambda}\right)
\end{array}\right)^{-1} \\
& \times\left(\begin{array}{cc}
1+\frac{\sinh [(\pi-\omega) \lambda]}{\sinh \pi \lambda} & 0 \\
0 & 1-\frac{\sinh [(\pi-\omega) \lambda]}{\sinh \pi \lambda}
\end{array}\right)\left(\begin{array}{l}
\hat{g}^{e}(\lambda-i) \\
\hat{g}^{o}(\lambda-i)
\end{array}\right) \\
& =\frac{1}{\lambda}\left(\begin{array}{cc}
\frac{\cosh (\omega / 2) \lambda}{\sinh (\omega / 2) \lambda} & 0 \\
0 & \frac{\sinh (\omega / 2) \lambda}{\cosh (\omega / 2) \lambda}
\end{array}\right)\left(\begin{array}{l}
\hat{g}^{e}(\lambda-i) \\
\hat{g}^{o}(\lambda-i)
\end{array}\right) .
\end{aligned}
$$

Comparing this transformed equation with (22) we detect a similar Mellin symbol with the factor $\lambda$ being replaced with $1 / \lambda$ and arguments of $\hat{v}$ and $\hat{g}$ being shifted by $+i$ and $-i$, respectively. Therefore, we conclude for $v^{e}$ and $v^{o}$ expansions of the form

$$
\begin{aligned}
v^{e}(x)= & \sum_{k=1}^{n}\left(v_{k}^{e, 1} x^{2 k(\pi / \omega)}+v_{k}^{e, 2} x^{2 k(\pi / \omega)} \log |x|\right) \\
& +\sum_{1 \leq k<2 n(\pi / \omega)} v_{k}^{e, 3} x^{k}+v_{0}^{e}(x)
\end{aligned}
$$


and

$$
\begin{aligned}
v^{o}(x)= & \sum_{k=0}^{n}\left(v_{k}^{o, 1} x^{(2 k+1)(\pi / \omega)}+v_{k}^{o, 2} x^{(2 k+1)(\pi / \omega)} \log |x|\right) \\
& +\sum_{1 \leq k<(2 n+1)(\pi / \omega)} v_{k}^{o, 3} x^{k}+v_{0}^{o}(x)
\end{aligned}
$$

where

$$
v_{0}^{e} \in \tilde{H}^{s}\left(\mathbf{R}_{+}\right) \quad \text { for } s-1 / 2 \in\left(2 n \frac{\pi}{\omega},\left[2 n \frac{\pi}{\omega}+1\right]\right)
$$

and

$$
v_{0}^{o} \in \tilde{H}^{s}\left(\mathbf{R}_{+}\right) \quad \text { for } s-1 / 2 \in\left((2 n+1) \frac{\pi}{\omega},\left[(2 n+1) \frac{\pi}{\omega}+1\right]\right)
$$

Analogously, as above, we obtain

$$
\left|v_{k}^{e, 1}\right|= \begin{cases}\frac{1}{k \pi}\left|\hat{g}^{e}\left(i\left(2 k \frac{\pi}{\omega}-1\right)\right)\right| & (2 k(\pi / \omega) \text { not an integer }) \\ \frac{1}{k \pi} \mid \hat{g}^{e}(\lambda-i) & \\ -\left.\frac{g_{2 k(\pi / \omega)-1}^{e}}{\lambda-i 2 k(\pi / \omega)}\right|_{\lambda=i 2 k(\pi / \omega)} & (2 k(\pi / \omega) \text { integer })\end{cases}
$$

$$
\left|v_{k}^{e, 2}\right|= \begin{cases}0 & (2 k(\pi / \omega) \text { not an integer }) \\ (1 /(k \pi))\left|g_{2 k(\pi / \omega)-1}^{e}\right| & (2 k(\pi / \omega) \text { integer })\end{cases}
$$

$$
\left|v_{k}^{e, 3}\right|= \begin{cases}\frac{1}{k}\left|\frac{\cos (\omega / 2) k}{\sin (\omega / 2) k}\right|\left|g_{k-1}^{e}\right| & (k \neq 2 m(\pi / \omega) \text { for all } \\ 0 & \text { integers } m) \\ 0 & \text { (else) }\end{cases}
$$


and

$$
\left|v_{k}^{o, 1}\right|= \begin{cases}\frac{2}{(2 k+1) \pi}\left|\hat{g}^{o}\left(i(2 k+1) \frac{\pi}{\omega}-i\right)\right| & ((2 k+1)(\pi / \omega) \text { not } \\ \frac{2}{(2 k+1) \pi} \mid \hat{g}^{o}(\lambda-i) & \\ -\frac{g_{(2 k+1)(\pi / \omega)-1}^{o}}{\lambda-i(2 k+1) \pi / \omega} \mid & ((2 k+1)(\pi / \omega) \text { integer })\end{cases}
$$$$
\left|v_{k}^{o, 2}\right|= \begin{cases}0 & ((2 k+1)(\pi / \omega) \text { not an integer }) \\ \frac{2}{(2 k+1) \pi}\left|g_{(2 k+1)(\pi / \omega)-1}^{o}\right| & ((2 k+1)(\pi / \omega) \text { integer })\end{cases}
$$

$$
\left|v_{k}^{o, 3}\right|=\left\{\begin{array}{ll}
\frac{1}{k}\left|\frac{\sin (\omega / 2) k}{\cos (\omega / 2) k}\right|\left|g_{k-1}^{o}\right| & (k \neq(2 m+1)(\pi / \omega) \text { for all integers } m) \\
0 & (\text { else })
\end{array} .\right.
$$

Now, mapping back to functions on $\Gamma^{-}$and $\Gamma^{+}$via the relations (14), i.e., $\left.\psi\right|_{\Gamma^{-}}=\psi_{-}=\psi^{e}+\psi^{o}$ and $\left.\psi\right|_{\Gamma^{+}}=\psi_{+}=\psi^{e}-\psi^{o}$, we obtain expansions of $\psi$ and $v$ on $\Gamma^{\omega}$ near the corner. Therefore, we proved the representations (9) and (10). For the coefficients there holds

$$
\begin{aligned}
c_{2 k+1}^{j, 1} & =\left(\begin{array}{c}
\psi_{k}^{e, 1} \\
\psi_{k}^{e, 1}
\end{array}\right), & c_{2 k+1}^{j, 2} & =\left(\begin{array}{c}
\psi_{k}^{e, 2} \\
\psi_{k}^{e, 2}
\end{array}\right) \\
c_{2 k}^{j, 1} & =\left(\begin{array}{c}
\psi_{k}^{o, 1} \\
-\psi_{k}^{o, 1}
\end{array}\right), & c_{2 k}^{j, 2} & =\left(\begin{array}{c}
\psi_{k}^{o, 2} \\
-\psi_{k}^{o, 2}
\end{array}\right)
\end{aligned}
$$

(cf. (26), (29), (27), (30)) and

$$
\begin{aligned}
d_{2 k+1}^{j, 1} & =\left(\begin{array}{c}
v_{k}^{o, 1} \\
-v_{k}^{o, 1}
\end{array}\right), & d_{2 k+1}^{j, 2} & =\left(\begin{array}{c}
v_{k}^{o, 2} \\
-v_{k}^{o, 2}
\end{array}\right) \\
d_{2 k}^{j, 1} & =\left(\begin{array}{c}
v_{k}^{e, 1} \\
v_{k}^{e, 1}
\end{array}\right), & d_{2 k}^{j, 2} & =\left(\begin{array}{c}
v_{k}^{e, 2} \\
v_{k}^{e, 2}
\end{array}\right)
\end{aligned}
$$

(cf. (34), (37), (35), (38)) where $\omega$ has to be replaced with $\omega_{j}$ and where, for $f^{e}, f^{o}$ and $g^{e}, g^{o}$ local mappings of the given data $f$ and $g$ 
onto $\Gamma^{\omega}$ have to be used. We note that we did not consider explicitly the smoothness of the solutions of the integral equations around the corners. This was done in detail by Costabel and Stephan in [3].

Since we are interested in the exact behaviors of the solutions of the integral equations, where the usual Sobolev spaces are not appropriate, we have to let $n$ in (9) and (10) tend to infinity. In view of the relations (26)-(31) and (34)-(39) and Lemma 4 we make the following assumptions to control the growth of the coefficients in the expansions (9) and (10) (cf. also (24), (25), (32), (33)):

Assumption 1. There exist constants $C$ and $\gamma$ such that, for $j=1, \ldots, J$ and for integers $k$ large enough,

$$
\begin{aligned}
&\left|\hat{f}\left(i k \frac{\pi}{\omega_{j}}\right)\right| \leq C^{k \gamma} \quad\left(k \frac{\pi}{\omega_{j}} \text { not an integer }\right) \\
& \mid \hat{f}(\lambda)-\frac{\left.f_{k\left(\pi / \omega_{j}\right)}\right|_{\lambda-i k\left(\pi / \omega_{j}\right)}}{\lambda=i k\left(\pi / \omega_{j}\right)} \leq C^{k \gamma} \quad\left(k \frac{\pi}{\omega_{j}} \text { integer }\right) \\
&\left|f_{k\left(\pi / \omega_{j}\right)}\right| \leq C^{k \gamma} \quad\left(k \frac{\pi}{\omega_{j}} \text { integer }\right) \\
&\left|\frac{f_{k}}{\sin \left(\omega_{j} / 2\right) k \cos \left(\omega_{j} / 2\right) k}\right| \leq C^{k \gamma} \\
&\left(k \neq m \frac{\pi}{\omega_{j}} \text { for all integers } m\right) .
\end{aligned}
$$

Assumption 2. There exist constants $C$ and $\gamma$ such that, for $j=1, \ldots, J$ and for integers $k$ large enough,

$$
\left|\hat{g}\left(i\left(k \frac{\pi}{\omega_{j}}-1\right)\right)\right| \leq C^{k \gamma} \quad\left(k \frac{\pi}{\omega_{j}} \text { not an integer }\right)
$$

$$
\begin{array}{r}
\left|\hat{g}(\lambda-i)-\frac{g_{k\left(\pi / \omega_{j}\right)-1}}{\lambda-i k\left(\pi / \omega_{j}\right)}\right|_{\lambda=i k\left(\pi / \omega_{j}\right)} \leq C^{k \gamma} \quad\left(k \frac{\pi}{\omega_{j}} \text { integer }\right) \\
\left|g_{k\left(\pi / \omega_{j}\right)-1}\right| \leq C^{k \gamma} \quad\left(k \frac{\pi}{\omega_{j}} \text { integer }\right)
\end{array}
$$




$$
\begin{aligned}
\left|\frac{g_{k-1}}{\sin \left(\omega_{j} / 2\right) k \cos \left(\omega_{j} / 2\right) k}\right| \leq C^{k \gamma} \\
\quad\left(k \neq m \frac{\pi}{\omega_{j}} \text { for all integers } m\right) .
\end{aligned}
$$

These assumptions are to be understood locally at each of the corners of $\Gamma$, i.e., by mapping $\Gamma_{j-1} \cup\{0\} \cup \Gamma_{j}$ onto $\Gamma^{\omega}$, where we made no differences between even and odd functions.

Under the two above assumptions, we deduce the following corollary which can be proved exactly by the steps performed in the proof of Theorem 1.

Corollary 1. (i) Provided $f$ is piecewise analytic and fulfills Assumption 1 then the solution $\psi$ of (3) has for any $x \in \Gamma$ the form

$$
\begin{gathered}
\psi(x)=\sum_{j=1}^{J} \sum_{k=1}^{\infty}\left(c_{k}^{j, 1}\left|x-x_{j}\right|^{\alpha_{k_{j}}-1}+c_{k}^{j, 2}\left|x-x_{j}\right|^{\alpha^{k_{j}}-1} \log \left|x-x_{j}\right|\right. \\
\left.+c_{k}^{j, 3}\left|x-x_{j}\right|^{k-1}\right) \chi_{j}(x)+\psi_{0}(x), \\
c_{k}^{j, 1}, c_{k}^{j, 2}, c_{k}^{j, 3} \in \mathbf{R}^{2}, \quad \alpha_{k j}=k\left(\pi / \omega_{j}\right)
\end{gathered}
$$

with $\left.\psi_{0}\right|_{\Gamma^{j}} \in C^{\infty}\left(\Gamma^{j}\right)$. For the components of the coefficients $c_{k}^{j, 1}, c_{k}^{j, 2}$ and $c_{k}^{j, 3}$ there holds

$$
\left|{ }^{ \pm} c_{k}^{j, 1}\right| \leq C_{1}^{k \gamma_{1}}, \quad\left|{ }^{ \pm} c_{k}^{j, 2}\right| \leq C_{1}^{k \gamma_{1}} \quad \text { and } \quad\left|{ }^{ \pm} c_{k}^{j, 3}\right| \leq C_{1}^{k \gamma_{1}} .
$$

The constants $C_{1}$ and $\gamma_{1}$ depend on $f$ and $\omega_{j}, j=1, \ldots, J$.

(ii) The solution $v$ of (4) for piecewise analytic $g$ fulfilling Assumption 2 has for any $x \in \Gamma$ the form

$$
\begin{aligned}
v(x)=\sum_{j=1}^{J} \sum_{k=1}^{\infty}\left(d_{k}^{j, 1}\left|x-x_{j}\right|^{\alpha_{k_{j}}}+d_{k}^{j, 2}\left|x-x_{j}\right|^{\alpha_{k_{j}}} \log \left|x-x_{j}\right|\right. \\
\left.+d_{k}^{j, 3}\left|x-x_{j}\right|^{k}\right) \chi_{j}(x)+v_{0}(x), \quad d_{k}^{j, 1}, d_{k}^{j, 2}, d_{k}^{j, 3} \in \mathbf{R}^{2}
\end{aligned}
$$


with $\left.v_{0}\right|_{\Gamma^{j}} \in C^{\infty}\left(\Gamma^{j}\right)$. For the components of the coefficients $d_{k}^{j, 1}, d_{k}^{j, 2}$ and $d_{k}^{j, 3}$ there holds

$$
\left|{ }^{ \pm} d_{k}^{j, 1}\right| \leq C_{2}^{k \gamma_{2}}, \quad\left|{ }^{ \pm} d_{k}^{j, 2}\right| \leq C_{2}^{k \gamma_{2}} \quad \text { and }\left.\quad\right|^{ \pm} d_{k}^{j, 3} \mid \leq C_{2}^{k \gamma_{2}} .
$$

The constants $C_{2}$ and $\gamma_{2}$ depend on $g$ and $\omega_{j}, j=1, \ldots, J$.

Here $\chi_{j}$ is a $C^{\infty}$ cut-off function concentrated at the jth corner $x_{j}$, with opening angle $\omega_{j}$.

If $f$ and $g$ have finite expansions of the form (23) at the corners of $\Gamma$, i.e. they are locally polynomials of arbitrary degree, the Assumptions 1 and 2 are automatically fulfilled.

Corollary 2. (i) Let $f$ be piecewise analytic and have polynomial behavior at the corners of $\Gamma$. Then, the solution $\psi$ of (3) has, for any $x \in \Gamma$, the form

$$
\begin{gathered}
\psi(x)=\sum_{j=1}^{J} \sum_{k=1}^{\infty}\left(c_{k}^{j, 1}\left|x-x_{j}\right|^{\alpha_{k j}-1}+c_{k}^{j, 2}\left|x-x_{j}\right|^{\alpha_{k j}-1} \log \left|x-x_{j}\right|\right) \\
\cdot \chi_{j}(x)+\psi_{0}(x), \quad c_{k}^{j, 1}, c_{k}^{j, 2} \in \mathbf{R}^{2}, \quad \alpha_{k j}=k \frac{k}{\omega_{j}}
\end{gathered}
$$

with $\left.\psi_{0}\right|_{\Gamma^{j}} \in C^{\infty}\left(\Gamma^{j}\right)$. For the components of the coefficients $c_{k}^{j, 1}$ and $c_{k}^{j, 2}$ there holds

$$
\left|{ }^{ \pm} c_{k}^{j, 1}\right| \leq C_{1}^{k_{\gamma_{1}}}
$$

and

$$
{ }^{ \pm} c_{k}^{j, 2}=0 \quad \text { for } k \geq k_{1}
$$

for constants $C_{1}, \gamma_{1}$ and an integer $k_{1}$ depending on $f$ and $\omega_{j}, j=$ $1, \ldots, J$.

(ii) Let $g$ be piecewise analytic and have polynomial behavior at the corners of $\Gamma$. Then, the solution $v$ of (4) has, for any $x \in \Gamma$, the form

$$
\begin{gathered}
v(x)=\sum_{j=1}^{J} \sum_{k=1}^{\infty}\left(d_{k}^{j, 1}\left|x-x_{j}\right|^{\alpha_{k j}}+d_{k}^{j, 2}\left|x-x_{j}\right|^{\alpha_{k j}} \log \left|x-x_{j}\right|\right) \\
\cdot \chi_{j}(x)+v_{0}(x), \quad d_{k}^{j, 1}, d_{k}^{j, 2} \in \mathbf{R}^{2},
\end{gathered}
$$


with $\left.v_{0}\right|_{\Gamma^{j}} \in C^{\infty}\left(\Gamma^{j}\right)$. For the components of the coefficients $d_{k}^{j, 1}$ and $d_{k}^{j, 2}$ there holds

$$
\left|{ }^{ \pm} d_{k}^{j, 1}\right| \leq C_{2}^{k_{\gamma_{2}}}
$$

and

$$
{ }^{ \pm} d_{k}^{j, 2}=0 \quad \text { for } k \geq k_{2} .
$$

The constants $C_{2}, \gamma_{2}$ and the integer $k_{2}$ depend on $g$ and $\omega_{j}, j=$ $1, \ldots, J$.

Here $\chi_{j}$ is a $C^{\infty}$ cut-off function concentrated at the $j$ th corner $x_{j}$, with opening angle $\omega_{j}$.

Proof. The assertion follows from Corollary 1 by noting that $c_{k}^{j, 3} \neq 0$ and $d_{k}^{j, 3} \neq 0$ only for finitely many numbers $k$ due to the polynomial behavior of $f$ and $g$ at the corners of $\Gamma$. Therefore, the corresponding terms in the expansion for $\psi$ are again polynomials and can be incorporated in the remainders $\psi_{0}$ and $v_{0}$. For the same reason, the local Mellin transforms of $f$ and $g$ possess only finitely many poles of second order which means that almost all of the coefficients $c_{k}^{j, 2}$ and $d_{k}^{j, 2}$ are zero. By noting that $f_{k}=0$ and $g_{k}=0$ for $k$ large enough (cf . (23)) and since therefore $\hat{f}$ and $\hat{g}$ have no poles at $i k\left(\pi / \omega_{j}\right), j=1, \ldots, J$, for $k$ large enough, a comparison of (26)-(31) and (34)-(39) with Assumptions 1 and 2 shows that only the growth conditions (42) and (43) have to be required. On the other hand, these growth conditions are automatically satisfied if $f$ and $g$ have polynomial behavior at the corners of $\Gamma$. This follows from the fact that the Mellin transform of smooth functions with compact support satisfy (42) and (43) due to $[\mathbf{5}$, Lemma $4.2,(4.7)]$. Note that we only consider the functions locally at the corners by use of appropriate $C^{\infty}$ cut-off functions.

Obviously, our method of using the Mellin transformation to obtain singular expansions of the solutions of the integral equations applies to more general given data $f$ and $g$ as well. What we basically need are expansions of $f$ and $g$ at the corners of $\Gamma$ which may also contain singular terms of the form $\left(x-x_{j}\right)^{\alpha}, \alpha \in \mathbf{R}$. In the following corollary, we consider functions $f$ and $g$ which have finitely many singular terms at the corners of $\Gamma$ which are treated explicitly by the Mellin transform. The remainders of $f$ and $g$ (after having considered the singular terms) 
are again arbitrarily smooth. Altogether the inspection of the proofs of the foregoing corollaries and Theorem 1 shows that there holds the following result (the proof is omitted for brevity).

Corollary 3. (i) Let $f$ be analytic on $\Gamma$ apart from the corners and suppose $f$ has expansions of the form

$$
f(x)=\sum_{k=1}^{n_{j}} f_{k}^{j}\left|x-x_{j}\right|^{\nu_{k j}}, \quad \nu_{k j} \in \mathbf{R}
$$

at the corners $x_{j}$ of $\Gamma$ with $\nu_{1 j}>\alpha_{1 j}, j=1, \ldots, J$ and $\alpha_{k j} \neq \nu_{m n}$ for all pairs $k j$ and $m n$. Then the solution $\psi$ of (3) has, for any $x \in \Gamma$, the form

$$
\begin{gathered}
\psi(x)=\sum_{j=1}^{J} \sum_{k=1}^{\infty}\left(c_{k}^{j, 1}\left|x-x_{j}\right|^{\alpha_{k j}-1}+c_{k}^{j, 3}\left|x-x_{j}\right|^{\nu_{k j}-1}\right) \chi_{j}(x)+\psi_{0}(x), \\
c_{k}^{j, 1}, c_{k}^{j, 3} \in \mathbf{R}^{2}, \quad \alpha_{k j}=k\left(\pi / \omega_{j}\right)
\end{gathered}
$$

with $\left.\psi_{0}\right|_{\Gamma^{j}} \in C^{\infty}\left(\Gamma^{j}\right)$. For the components of the coefficients $c_{k}^{j, 1}$ and $c_{k}^{j, 3}$ there holds

$$
\left|{ }^{ \pm} c_{k}^{j, 1}\right| \leq C_{1}^{k \gamma_{1}}
$$

and

$$
{ }^{ \pm} c_{k}^{j, 3}=0 \quad \text { for } k>n_{j}
$$

for constants $C_{1}$ and $\gamma_{1}$ depending on $f$ and $\omega_{j}, j=1, \ldots, J$.

(ii) Let $g$ be analytic on $\Gamma$ apart from the corners and suppose $g$ has expansions of the form

$$
g(x)=\sum_{k=1}^{n_{j}} g_{k}^{j}\left|x-x_{j}\right|^{\nu_{k j}-1}
$$

at the corners $x_{j}$ of $\Gamma$. Then the solution $v$ of (4) has, for any $x \in \Gamma$, the form

$$
\begin{gathered}
v(x)=\sum_{j=1}^{J} \sum_{k=1}^{\infty}\left(d_{k}^{j, 1}\left|x-x_{j}\right|^{\alpha_{k j}}+d_{k}^{j, 3}\left|x-x_{j}\right|^{\nu_{k j}}\right) \chi_{j}(x)+v_{0}(x), \\
d_{k}^{j, 1}, d_{k}^{j, 3} \in \mathbf{R}^{2}
\end{gathered}
$$


with $\left.v_{0}\right|_{\Gamma^{j}} \in C^{\infty}\left(\Gamma^{j}\right)$. For the components of the coefficients $d_{k}^{j, 1}$ and $d_{k}^{j, 3}$ there holds

$$
\left|{ }^{ \pm} d_{k}^{j, 1}\right| \leq C_{2}^{k \gamma_{2}}
$$

and

$$
{ }^{ \pm} d_{k}^{j, 3}=0 \quad \text { for } k>n_{j} .
$$

The constants $C_{2}$ and $\gamma_{2}$ depend on $g$ and $\omega_{j}, j=1, \ldots, J$.

Here $\chi_{j}$ is a $C^{\infty}$ cut-off function concentrated at the $j$ th corner $x_{j}$, with opening angle $\omega_{j}$.

Remark 2. If some of the exponents $\alpha_{k j}$ and $\nu_{m n}$ are identical, there occur also logarithmic terms, as in the previous corollaries.

4. The hp-version of the boundary element method. To describe the hp-version we introduce the geometric mesh $\Gamma_{\sigma}^{n}$ on $\Gamma=$ $\cup_{j=1}^{J} \Gamma^{j}, \Gamma^{j}$ being open arcs, with endpoints $x_{j-1}, x_{j}$. First, we bisect each side $\Gamma^{j}$ with length $d_{j}$ into two pieces $\Gamma_{1}^{j}$ (containing the vertex $x_{j-1}$ ) and $\Gamma_{2}^{j}$ (containing the vertex $x_{j}$ ). Then each boundary piece $\Gamma_{k}^{j}, j=1, \ldots, J, k=1,2$, is decomposed into subarcs $\Gamma_{k}^{j, m}, m=$ $1, \ldots, n+1$, geometrically refined towards the vertices $x_{j-2+k}$,

$$
\operatorname{dist}\left(x_{j-2+k}, \Gamma_{k}^{j, m+1}\right)=\left(d_{j} / 2\right) \sigma^{n-m+1}, \quad m=1, \ldots, n,
$$

where $\sigma \in(0,1)$ is the mesh grading parameter and $n+1$ is the number of levels of the mesh, cf. Figure 2 in Section 6 where the mesh is geometrically graded just towards the origin. On this geometric mesh $\Gamma_{\sigma}^{n}$ the boundary element space $S^{P, l}\left(\Gamma_{\sigma}^{n}\right), l=0$ or 1 , is given by

$$
\begin{aligned}
S^{P, l}\left(\Gamma_{\sigma}^{n}\right):=\left\{\psi \in H^{l}(\Gamma) ;\left.\psi\right|_{\Gamma_{k}^{j, m}} \in P_{p_{k}^{j, m}}\left(\Gamma_{k}^{j, m}\right),\right. & \\
& j=1, \ldots, J, k=1,2, m=1, \ldots, n+1\}
\end{aligned}
$$

where $P_{p}\left(\Gamma_{k}^{j, m}\right)$ denotes the space of polynomials of degree $\leq p$ on the subarc $\Gamma_{k}^{j, m}$.

With the choice $X_{N}:=S^{P, 0}\left(\Gamma_{\sigma}^{n}\right)$ in the Galerkin scheme (5), we have

Theorem 2. Provided the given data $f$ in (1) satisfies the assumptions of one of the corollaries 1(i), 2(i) and 3(i), then there holds the 
estimate

$$
\left\|\psi-\psi_{N}\right\|_{H^{-1 / 2}(\Gamma)} \leq C e^{-b \sqrt{N}}
$$

for the error between the Galerkin solution $\psi_{N} \in S^{P, 0}\left(\Gamma_{\sigma}^{n}\right)$ of (5) and the solution $\psi$ of $(3)$ if the degrees $P$ are suitably chosen. Here the positive constants $C$ and $b$ depend on the mesh parameter $\sigma$ but not on the dimension $N$ of $S^{P, 0}\left(\Gamma_{\sigma}^{n}\right)$.

With the choice $Y_{N}:=S^{P, 1}\left(\Gamma_{\sigma}^{n}\right)$ in the Galerkin scheme (6) we have

Theorem 3. Provided the given data $g$ in (2) satisfies the assumptions of one of the corollaries 1(ii), 2(ii) and 3(ii), then there holds the estimate

$$
\left\|v-v_{N}\right\|_{H^{1 / 2}(\Gamma)} \leq C e^{-b \sqrt{N}}
$$

for the error between the Galerkin solution $v_{N} \in S^{P, 1}\left(\Gamma_{\sigma}^{n}\right)$ of (6) and the solution $v$ of (4) if the degrees $P$ are suitably chosen. Here the positive constants $C$ and $b$ depend on $\sigma$ but are independent of $N=\operatorname{dim} S^{P, 1}\left(\Gamma_{\sigma}^{n}\right)$.

Remark 3. The functions in $X_{N}$ need not be continuous on $\Gamma$ since $X_{N} \subset H^{-1 / 2}(\Gamma)$ whereas the constraint $Y_{N} \subset H^{1 / 2}(\Gamma)$ requires continuity for the functions in $Y_{N}$.

The proofs of Theorems 2 and 3 are based on the regularity results for the solutions of the integral equations presented in Section 3 and on approximation results for splines on geometric meshes.

Following Guo and Babuška, see e.g. [7], we shall study the approximation of singular functions which can be characterized as elements of special Sobolev spaces, the countably normed spaces, which will be introduced in the following.

Let $I=(0,1)$. By $H_{\beta}^{m, l}(I), m \geq l \geq 1$ integers, we denote the completion of the set of all infinitely differentiable functions under the norm

$$
\|u\|_{H_{\beta}^{m, l}(I)}^{2}=\|u\|_{H^{l-1}(I)}^{2}+\sum_{k=l}^{m}\left\|u^{(k)} x^{(\beta+k-l)}\right\|_{L^{2}(I)}^{2} .
$$


The countably normed spaces on $I$ are defined as

$$
\begin{gathered}
B_{\beta}^{l}(I)=\left\{u \in H_{\beta}^{m, l}(I), m=l, l+1, \ldots ; \exists C \geq 0, d \geq 1, k=l, l+1, \ldots\right. \\
\left.\left\|u^{(k)} x^{(\beta+k-l)}\right\|_{L^{2}(I)} \leq C d^{(k-l)}(k-l) !\right\} \quad l \geq 1, \text { integer. }
\end{gathered}
$$

On $\Gamma$ the countably normed spaces are the product spaces

$$
B_{\beta}^{l}(\Gamma)=\Pi_{j=1}^{J} \Pi_{k=1}^{2} B_{\beta}^{l}\left(\Gamma_{k}^{j}\right) \cap H^{l-1}(\Gamma)
$$

where each boundary piece $\Gamma_{k}^{j}$ has to be mapped onto $I$ such that the vertices $x_{j+k-2}$ fall onto 0 in order to apply the definition (48).

For the local singularity terms, we have

Lemma 3. Let $R>0$ and $\varphi_{\mu}(x):=x^{\mu}, \varphi_{\mu, k}(x):=x^{\mu} \log ^{k} x$ for $x \in(0, R), k>0$ an integer. Then

(i) $\varphi_{\mu} \in B_{\beta}^{l}(0, R)$ for $\mu>l-1 / 2-\beta$,

(ii) $\varphi_{\mu, k} \in B_{\beta}^{l}(0, R)$ for $\mu>l-1 / 2-\beta$.

The proof of Lemma 3 follows immediately by inspection.

Lemma 4. Let

$$
\varphi(x):=\sum_{n=1}^{\infty} c_{n} x^{n(\pi / \omega)+l-2} \in H^{l-1}\left(0, R^{\prime}\right)
$$

$(l=1,2)$ with $\left|c_{n}\right| \leq C^{n \gamma}$ for constants $C$ and $\gamma$. Then there holds

$$
\varphi \in B_{\beta}^{l}(0, R)
$$

for all $\beta \in(3 / 2-\pi / \omega, 1)$ and $R \leq R^{\prime}$ small enough. 
Proof. We have to show $\left\|\varphi^{(k)} x^{\beta+k-l}\right\|_{L^{2}(0, R)} \leq C d^{k-l}(k-l) !, k \geq l$. With $\alpha_{n}=n(\pi / \omega)+l-2$ and $(\alpha)_{k}:=\alpha(\alpha-1) \cdots(\alpha-k+1)$, we have

$$
\begin{aligned}
\left\|x^{\beta+k-l} \varphi^{(k)}\right\|_{L^{2}(0, R)}^{2} & =\int_{0}^{R}\left|\varphi^{(k)}(x)\right|^{2} x^{2(\beta+k-l)} d x \\
& =\int_{0}^{R}\left|\sum_{n=1}^{\infty} c_{n}\left(\alpha_{n}\right)_{k} x^{\alpha_{n}+\beta-2}\right|^{2} d x \\
& =\int_{0}^{R}\left|x^{\alpha_{1}+\beta-2}\right|^{2}\left|\sum_{n=1}^{\infty} c_{n}\left(\alpha_{n}\right)_{k} x^{(n-1)(\pi / \omega)}\right|^{2} d x .
\end{aligned}
$$

Now the series is bounded, provided $R$ is small enough: Let $K$ be the integer defined by $\left(\alpha_{K}\right)_{k}<0,\left(\alpha_{K+1}\right)_{k}>0$. Then we conclude

$$
\begin{aligned}
\left|\sum_{n=1}^{\infty} c_{n}\left(\alpha_{n}\right)_{k} x^{(n-1)(\pi / \omega)}\right| \leq & \sum_{n=1}^{\infty}\left|c_{n}\right|\left|\left(\alpha_{n}\right)_{k}\right| R^{(n-1)(\pi / \omega)} \\
= & \sum_{n=1}^{K}\left|c_{n}\right|\left|\left(\alpha_{n}\right)_{k}\right| R^{(n-1)(\pi / \omega)} \\
& +\sum_{n=K+1}^{\infty}\left|c_{n}\right|\left|\left(\alpha_{n}\right)_{k}\right| R^{(n-1)(\pi / \omega)} \\
\leq & c k ! \sum_{n=1}^{K}\left|c_{n}\right| R^{(n-1)(\pi / \omega)} \\
& +c(\pi / \omega)^{k} \sum_{n=K+1}^{\infty}\left|c_{n}\right| n^{k} R^{(n-1)(\pi / \omega)} \\
\leq & c k !+c(\pi / \omega)^{k} \\
\leq & c d^{k-l}(k-l) !
\end{aligned}
$$

for $R$ small enough due to the assumption $\left|c_{n}\right| \leq C^{n \gamma}$. Here we have used

$$
\left|\left(\alpha_{n}\right)_{k}\right| \leq \begin{cases}\left(\alpha_{n}\right)^{k} & \text { for } \alpha_{n} \geq k \\ \Gamma\left(\alpha_{n}+1\right) \Gamma\left(k-\alpha_{n}\right) & \text { for } \alpha_{n}<k\end{cases}
$$

and

$$
\Gamma\left(\alpha_{n}+1\right) \Gamma\left(k-\alpha_{n}\right) \leq c k ! \text { for } \alpha_{n}<k .
$$


Combining (50) and (51), we obtain for $3 / 2-\pi / \omega<\beta<1$,

$$
\begin{aligned}
\left\|x^{\beta+k-l} \varphi^{(k)}\right\|_{L^{2}(0, R)} & \leq c d^{k-l}(k-l) !\left(\int_{0}^{R}\left|x^{(\pi / \omega)+\beta-2}\right|^{2} d x\right)^{1 / 2} \\
& \leq c d^{k-l}(k-l) !
\end{aligned}
$$

Remark 4. Inspection of the proof of Lemma 4 shows also that expansions of the form

$$
\sum_{n=1}^{\infty}\left(c_{n}^{1} x^{n \pi / \omega+l-2}+c_{n}^{2} x^{n \pi / \omega+l-2} \log |x|+c_{n}^{3} x^{n}\right)
$$

(given by Corollaries 1 and 2) and also that given by Corollary 3 are contained in $B_{\beta}^{l}(0, R)$ for $\beta>3 / 2-\pi / \omega$ and $R$ small enough.

Hence, if $f$ satisfies the assumptions of one of the Corollaries 1(i)-3(i), then for the solution $\psi$ of (3) there holds

$$
\psi-\psi_{0} \in B_{\beta}^{1}\left(U_{\delta}\left(x_{j}\right)\right) \quad \text { for } \beta>3 / 2-\pi / \omega_{j}
$$

for a neighborhood $U_{\delta}\left(x_{j}\right)$ of $x_{j}, j=1, \ldots, J$. Here $\psi_{0}$ is the $C^{\infty}$ remainder of the expansion of $\psi$ given by Corollaries 1(i)-3(i). Since $\psi=\left.(\partial u / \partial n)\right|_{\Gamma}$ in (3), $\psi$ is analytic apart from the corners for piecewise analytic $f$, and since $\Gamma$ can be covered by a suitable partition of unity we can conclude the following regularity on each of the sides of the polygon

$$
\begin{aligned}
\psi-\tilde{\psi}_{0} \in B_{\beta}^{1}\left(\Gamma_{k}^{j}\right) \quad \text { for } \beta & >\frac{3}{2}-\frac{\pi}{\omega_{j-2+k}} \\
j=1, \ldots, J, \quad k & =1,2 .
\end{aligned}
$$

Here $\tilde{\psi}_{0}$ consists of the $C^{\infty}$-remainder term $\psi_{0}$ in the expansion of $\psi$ and of the contributions from the expansions of $\psi$ which are localized away from the corners due to the partition of unity. Hence, $\tilde{\psi}_{0}$ is arbitrarily smooth on the sides of the polygon, i.e.,

$$
\left.\tilde{\psi}_{0}\right|_{\Gamma^{j}} \in C^{\infty}\left(\Gamma^{j}\right) .
$$


Analogously, if $g$ satisfies the assumptions of one of the Corollaries 1(ii) $-3($ ii), then for the solution $v$ of (4) there holds

$$
\begin{gathered}
v-\tilde{v}_{0} \in B_{\beta}^{2}\left(\Gamma_{k}^{j}\right) \quad \text { for } \beta>\frac{3}{2}-\frac{\pi}{\omega_{j-2+k}} \\
j=1, \ldots, J, \quad k=1,2
\end{gathered}
$$

since $v=\left.u\right|_{\Gamma}$ in (4) is analytic apart from the corners for piecewise analytic $g$. As above, $\tilde{v}_{0}$ consists of the $C^{\infty}$-remainder term $v_{0}$ in the expansion of $v$ and of the contributions from the expansions of $v$ which are localized away from the corners due to the partition of unity. Hence, $\tilde{v}_{0}$ is arbitrarily smooth on the sides of the polygon, i.e.,

$$
\left.\tilde{v}_{0}\right|_{\Gamma^{j}} \in C^{\infty}\left(\Gamma^{j}\right) .
$$

Next we need some properties of the Legendre polynomials.

Lemma 5. (i) Let $I=(-1,1), u(x)=\sum_{j=0}^{\infty} c_{j} l_{j}(x), l_{j}$ Legendre polynomial of degree $j$. Then

$$
\int_{I}\left|u^{(k)}(x)\right|^{2}\left(1-x^{2}\right)^{k} d x=\sum_{j \geq k} c_{j}^{2} \frac{2}{2 j+1} \frac{(j+k) !}{(j-k) !} .
$$

(ii) Let $I=(-1,1)$ and $u \in H^{k+1}(I), k \in \mathbf{N}_{0}$. Then there exist a $\varphi \in P_{k}(I)$ and a constant $c>0$ such that

$$
\left\|(u-\varphi)^{(m)}\right\|_{L^{2}(I)}^{2} \leq C \frac{(k-s) !}{(k+s+2-2 m) !}\left\|u^{(s+1)}\right\|_{L^{2}(I)}^{2}
$$

$\left(m=0,1,0 \leq s \leq k, s \in \mathbf{N}_{0}, k>0\right.$ or $\left.m=s=k=0\right)$ and $\varphi(-1)=u(-1), \varphi(1)=u(1)$ for $k>0$.

(iii) Let $J=(a, b), h=b-a$ and $u \in H^{k+1}(J), k \in \mathbf{N}_{0}$. Then there exist $a \varphi \in P_{k}(J)$ and a constant $C>0$ such that

$$
\begin{aligned}
& \left\|(u-\varphi)^{(m)}\right\|_{L^{2}(J)}^{2} \leq C h^{-2 m}\left(\frac{h}{2}\right)^{2(s+1)} \frac{(k-s) !}{(k+s+2-2 m) !}\left\|u^{(s+1)}\right\|_{L^{2}(J)}^{2} \\
& (m=0,1,0 \leq s \leq k, k>0 \text { or } m=s=k=0) \text { and } \varphi(a)=u(a), \\
& \varphi(b)=u(b) \text { for } k>0 .
\end{aligned}
$$


(iv) Let $I=(0, R)$ for $R>0, J=(a, b), J \subset I$ and $\lambda>0$ be $a$ fixed number with $h=b-a \leq \lambda a$. Then for $u \in H_{\beta}^{k+1, l}(I)$ there exist a polynomial $\varphi \in P_{k}(J)$ and a constant $c>0$ such that, for $m=0$ $(k=0)$ and $m=0,1(k>0)$, respectively, there holds

$$
\begin{aligned}
& \left\|(u-\varphi)^{(m)}\right\|_{L^{2}(J)}^{2} \leq C a^{2(l-m-\beta)} \\
& \frac{\Gamma(k-s+1)}{\Gamma(k+s+3-2 m)}\left(\frac{\lambda}{2}\right)^{2 s}|u|_{H_{\beta}^{s+1, l}(I)}^{2}
\end{aligned}
$$

$(m<s+1,1 \leq l \leq s+1 \leq k+1, s \in \mathbf{R})$ with $\varphi(a)=u(a), \varphi(b)=u(b)$ for $k>0$.

Proof. Assertion (i) is well-known (see, e.g., [7]). (ii) follows from (i) by expanding $u$ and $u^{\prime}$ in Legendre series (see [8]). (iii) follows from (ii) via affine transformation (see [8]). Assertion (iv) can be seen as follows. By definition,

$$
|u|_{H_{\beta}^{s+1, l}(I)}^{2} \geq a^{2(\beta+s+1-l)}\left\|u^{(s+1)}\right\|_{L^{2}(J)}^{2} .
$$

By (iii) there exists $\varphi \in P_{k}(J)$ with

$$
\begin{aligned}
\left\|(u-\varphi)^{(m)}\right\|_{L^{2}(J)}^{2} \leq C h^{-2 m} & \frac{(k-s) !}{(k+s+2-2 m) !} \\
& \left(\frac{h}{2}\right)^{2(s+1)} a^{-2(\beta+s+1-l)}|u|_{H_{\beta}^{s+1, l}(I)}^{2}
\end{aligned}
$$

yielding (iv).

Next we consider a geometric mesh $I_{\sigma}^{n}$ on $I=(0,1)$ with $n+1$ subintervals $I_{j}=\left[x_{j-1}, x_{j}\right], x_{0}=0, x_{j}=\sigma^{n-j+1}, h_{j}=x_{j}-x_{j-1}$, $1 \leq j \leq n+1$. For a degree vector $P=\left(p_{1}, \ldots, p_{n}\right)$ of nonnegative integers, we set

$$
S^{P, l}\left(I_{\sigma}^{n}\right):=\left\{q \in H^{l}(I) ;\left.q\right|_{I_{j}} \in P_{p_{j}}\left(I_{j}\right)\right\} .
$$

Lemma 6. Let $I=(0,1), u \in B_{\beta}^{l}(I), 0<\beta<1, l=1,2$. Then there exists a $\varphi \in S^{p, l-1}\left(I_{\sigma}^{n}\right)$ with $0<\sigma<1, p_{1}=l-1, p_{i}=\max \{l,[\mu i]\}$, $i=2, \ldots, n+1$, such that

$$
\|u-\varphi\|_{H^{l-1}(I)} \leq C e^{-b \sqrt{N}}
$$


where the positive constants $C$ and $b$ depend on $\sigma$ but are independent of $N=\operatorname{dim} S^{p, l-1}\left(I_{\sigma}^{n}\right)$.

Proof. First we use Lemma 5 (iv) on each subinterval $I_{i}, i>1$. Thus we have a $\varphi_{i} \in P_{p_{i}}\left(I_{i}\right)$ with

$$
\begin{aligned}
& \left\|\left(u-\varphi_{i}\right)^{(m)}\right\|_{L^{2}\left(I_{i}\right)}^{2} \leq C x_{i-1}^{2(l-m-\beta)} \\
& \qquad \frac{\Gamma\left(p_{i}-s_{i}+1\right)}{\Gamma\left(p_{i}+s_{i}+3-2 m\right)}\left(\frac{\lambda}{2}\right)^{2 s_{i}}|u|_{H_{\beta}^{s_{i}+1, l}(I)}^{2}
\end{aligned}
$$

$\left(m<s_{i}+1,1 \leq l \leq s_{i}+1 \leq p_{i}+1, s_{i} \in \mathbf{R}\right)$ since $u \in B_{\beta}^{l}(I)$ implies $u \in H_{\beta}^{s_{i}+1, l}(I), s_{i}+1 \geq l$. On the first interval $I_{i}, i=1$, we have (see [8])

$$
|| u-\left.\varphi_{1}\right|_{H^{l-1}\left(I_{1}\right)} ^{2} \leq C h_{1}^{2(1-\beta)}|u|_{H_{\beta}^{l, l}\left(I_{1}\right)}^{2} .
$$

Thus there exists $\varphi \in S^{p, l-1}\left(I_{\sigma}^{n}\right)$ with

$$
\begin{aligned}
&\|u-\varphi\|_{H^{l-1}(I)}^{2} \leq C\left[\sigma^{2(1-\beta) n}+\sum_{i=2}^{n+1} x_{i-1}^{2(1-\beta)}\right. \\
&\left.\frac{\Gamma\left(p_{i}-s_{i}+1\right)}{\Gamma\left(p_{i}+s_{i}+5-2 l\right)}\left(\frac{\lambda}{2}\right)^{2 s_{i}}|u|_{H_{\beta}^{s_{i}+1, l}(I)}^{2}\right] .
\end{aligned}
$$

With the estimate

$$
|u|_{H_{\beta}^{s+1, l}(I)} \leq C(l) d^{s} \Gamma(s+1), \quad s \in \mathbf{R}_{+}
$$

and

$$
x_{i}-x_{i-1} \leq \lambda x_{i-1}=\frac{1-\sigma}{\sigma} \sigma^{n-i+2}, \quad 2 \leq i \leq n+1
$$

we obtain

$$
\begin{aligned}
\|u-\varphi\|_{H^{l-1}(I)}^{2} \leq C\left[\sigma^{2(1-\beta) n}+\sum_{i=2}^{n+1} \sigma^{2(n-i+2)(1-\beta)}\right. \\
\left.\frac{\Gamma\left(p_{i}-s_{i}+1\right)}{\Gamma\left(p_{i}+s_{i}+5-2 l\right)} \Gamma\left(s_{i}+1\right)^{2}\left(\frac{\rho d}{2}\right)^{2 s_{i}}\right] \\
\leq C\left[\sigma^{2(1-\beta) n}+\sum_{i=2}^{n+1} \sigma^{2(n-i+2)(1-\beta)} p_{i}\left(F\left(\rho d, \alpha_{i}\right)\right)^{p_{i}}\right]
\end{aligned}
$$


where

$$
F(d, \alpha):=\left(\frac{\alpha d}{2}\right)^{2 \alpha} \frac{(1-\alpha)^{1-\alpha}}{(1+\alpha)^{1+\alpha}}
$$

and

$$
\alpha_{i}:=\max \left\{\frac{1}{p_{i}}, \alpha_{\min }\right\}, \quad \rho:=\max \{1, \lambda\} .
$$

There holds

$$
\inf _{\alpha \in(0,1)} F(d, \alpha)=F_{\min }=F\left(d, \alpha_{\min }\right)<1
$$

where

$$
\alpha_{\min }=\frac{2}{\sqrt{4+d^{2}}} .
$$

Taking $p_{i}=\max \{l,[\mu i]\}, i=2, \ldots, n+1([x]$ means the smallest integer greater or equal to $x$ ) with

$$
\mu>\max \left\{1, \frac{2(1-\beta) \log \sigma}{\log F_{\min }}\right\}
$$

and defining $i_{0}$ by $p_{i_{0}}=\left[1 / \alpha_{\min }\right]+1$, then $p_{i_{0}}=\left[\mu i_{0}\right] \leq 1 / \alpha_{\min }+2$ and thus $i_{0}$ is bounded. Hence

$$
\begin{gathered}
\|u-\varphi\|_{H^{l-1}(I)}^{2} \leq C\left[\sigma^{2(1-\beta) n}+\sum_{i=2}^{i_{0}} \sigma^{2(n-i+2)(1-\beta)} p_{i} F\left(\rho d, \alpha_{i}\right)^{p_{i}}\right. \\
\left.+\sum_{i=i_{0}+1}^{n+1} \sigma^{2(n-i+2)(1-\beta)} p_{i}\left(F_{\min }\right)^{p_{i}}\right] \\
\leq C \sigma^{2(1-\beta) n}\left[1+\sum_{i=2}^{i_{0}} \sigma^{2(2-i)(1-\beta)}\left(F_{\min }\right)^{p_{i}} p_{i}\right. \\
\cdot \max _{1 \leq i \leq i_{0}}\left(\frac{F\left(\rho d, 1 / p_{i}\right)}{F_{\min }}\right)^{p_{i}} \\
\left.+\sum_{i=i_{0}+1}^{n+1} \sigma^{2(2-i)(1-\beta)} p_{i}\left(F_{\min }\right)^{p_{i}}\right] .
\end{gathered}
$$


With $p_{i}=[\mu i]$ and $q:=F_{\min }^{\mu} / \sigma^{2(1-\beta)}<1$ due to (56) we have $\sum_{i>i_{0}} i q^{i}<\infty$ since $\left(i q^{i}\right)^{1 / i} \rightarrow q<1$ as $i \rightarrow \infty$. Therefore the term in the brackets is bounded yielding with a positive constant $c$

$$
\|u-\varphi\|_{H^{l-1}(I)}^{2} \leq c \sigma^{2(1-\beta) n} .
$$

Next we observe for $l=1$ :

$$
\begin{aligned}
N & =\operatorname{dim} S^{P, 0}\left(I_{\sigma}^{n}\right)=1+\sum_{i=2}^{n+1}\left(p_{i}+1\right) \\
& =1+\sum_{i=2}^{n+1}([\mu i]+1) \leq c \mu n^{2}
\end{aligned}
$$

and for $l=2$ :

$$
N=\operatorname{dim} S^{P, 1}\left(I_{\sigma}^{n}\right)=2+\sum_{i=2}^{n+1}\left(p_{i}+1\right)-n+2 \leq c \mu n^{2} .
$$

Hence we obtain from $(57)(l=1,2)$

$$
\|u-\varphi\|_{H^{l-1}(I)} \leq C e^{-b \sqrt{N}}
$$

with

$$
b=\frac{1-\beta}{\sqrt{\mu}} \log \frac{1}{\sigma} .
$$

Corollary 4. Let $I=(0,1), u \in B_{\beta}^{2}(I)$ for some $0<\beta<1$. Then there exists a $\varphi \in S^{P, 1}\left(I_{\sigma}^{n}\right)$ with $0<\sigma<1, p_{1}=1, p_{i}=[\mu i]$, $2 \leq i \leq n+1$, such that

$$
\|u-\varphi\|_{H^{1 / 2}(I)} \leq c e^{-b \sqrt{N}}
$$

with constants $c, b>0$ independent of $N=\operatorname{dim} S^{P, 1}\left(I_{\sigma}^{n}\right)$.

Proof. The assertion follows by interpolation directly from Lemma 6 .

$\square$ 
Now the proofs of Theorems 2 and 3 are completed as follows:

Proof of Theorem 3. First we observe that the assumptions on $g$ imply for the function $v$ satisfying (4) the representation $v=\tilde{v}+\tilde{v}_{0}$ where $\tilde{v} \in B_{\beta}^{2}\left(\Gamma_{k}^{j}\right)$ for $1>\beta>3 / 2-\pi / \omega_{j-2+k}$ and where $\tilde{v}_{0}$ is arbitrarily smooth, cf. (53). The $C^{\infty}$-part $\tilde{v}_{0}$ of $v$ can be approximated exponentially well as shown in [9]. For he part $\tilde{v}$ of $v$ we proceed as follows. By Lemma 6 there exists for each boundary piece $\Gamma_{k}^{j}$ a $w_{k}^{j} \in S^{P_{j, k}, 1}\left(\Gamma_{k}^{j}\right)$ with degree $p_{j, k, m}$ on $\Gamma_{k}^{j, m}$ such that $(l=1$ or 2$)$

$$
\left\|\tilde{v}-w_{k}^{j}\right\|_{H^{l-1}\left(\Gamma_{k}^{j}\right)} \leq C e^{-b_{j, k} \sqrt{N_{j, k}}}
$$

$N_{j, k}=\operatorname{dim} S^{P_{j, k}, 1}\left(\Gamma_{k}^{j}\right), k=1,2, j=1, \ldots, J$ where $w_{k}^{j}$ coincides with $\tilde{v}$ at the endpoints of $\Gamma_{k}^{j}$. Let

$$
\tilde{w}_{k}^{j}=\left\{\begin{array}{ll}
w_{k}^{j} & \text { on } \Gamma_{k}^{j} \\
0 & \text { elsewhere }
\end{array} \quad \text { and } \quad v_{k}^{j}= \begin{cases}\tilde{v} & \text { on } \Gamma_{k}^{j} \\
0 & \text { elsewhere. }\end{cases}\right.
$$

Then for $l=1$ and 2

$$
\begin{aligned}
\left\|\tilde{v}-\sum_{j=1}^{J} \sum_{k=1}^{2} \tilde{w}_{k}^{j}\right\|_{H^{l-1}(\Gamma)} & \leq \sum_{j=1}^{J} \sum_{k=1}^{2}\left\|v_{k}^{j}-\tilde{w}_{k}^{j}\right\|_{H^{l-1}(\Gamma)} \\
& =\sum_{j=1}^{J} \sum_{k=1}^{2}\left\|\left.\tilde{v}\right|_{\Gamma_{k}^{j}}-w_{k}^{j}\right\|_{H^{l-1}\left(\Gamma_{k}^{j}\right)} \\
& \leq C e^{-b \sqrt{N}}
\end{aligned}
$$

with $b=\min _{1 \leq j \leq J, 1 \leq k \leq 2}\left\{b_{j, k}\right\}, N=\min _{1 \leq j \leq J, 1 \leq k \leq 2}\left\{N_{j, k}\right\}$. Note the estimate (59) holds since $v_{k}^{j}-\tilde{w}_{k}^{j} \in C^{0}(\Gamma)$ and $v_{k}^{j}-\tilde{w}_{k}^{j} \equiv 0$ on $\Gamma \backslash \Gamma_{k}^{j}$. Hence the assertion of Theorem 3 follows from (59) by interpolation and by applying the right triangle inequality to $\| \tilde{v}+\tilde{v}_{0}-\sum_{j=1}^{J} \sum_{k=1}^{2} \tilde{w}_{k}^{j}-$ $\tilde{w}_{0} \|_{H^{1 / 2}(\Gamma)}$ where $\tilde{w}_{0}$ is a piecewise polynomial on the geometric mesh approximating $\tilde{v}_{0}$ sufficiently well (cf. [9]).

Proof of Theorem 2. First we observe that the assumptions on $f$ imply for the solution $\psi$ of (3) a representation of the form $\psi=\tilde{\psi}+\tilde{\psi}_{0}$ where $\tilde{\psi} \in B_{\beta}^{1}\left(\Gamma_{k}^{j}\right)$ for $1>\beta>3 / 2-\pi / \omega_{j-2+k}$ and where $\tilde{\psi}_{0}$ is arbitrarily 
smooth, cf. (52). Hence by Lemma 6 there exists for each boundary piece $\Gamma_{k}^{j}$ a $\varphi_{k}^{j} \in S^{P_{j, k}, 0}\left(\Gamma_{k}^{j}\right)$ with degree $p_{j, k, m}-1$ on $\Gamma_{k}^{j, m}$ such that

$$
\left\|\tilde{\psi}-\varphi_{k}^{j}\right\|_{L^{2}\left(\Gamma_{k}^{j}\right)} \leq C e^{-b_{j, k} \sqrt{N_{j, k}}}, \quad N_{j, k}=\operatorname{dim} S^{P_{j, k}, 0}\left(\Gamma_{j}^{k}\right) .
$$

Hence the assertion of Theorem 2 follows as in the proof of Theorem 3.

口

5. Applications to acoustic scattering. We consider for $\mu, k_{1}, k_{2} \in \mathbf{C} \backslash\{0\}$ and $\mu \neq-1$ the transmission problem

$$
\left.\begin{array}{llrl}
\left(\Delta+k_{1}^{2}\right) u_{1}=0 & \text { in } \Omega_{1} & u_{1}=u_{2}+v_{0} \\
\left(\Delta+k_{1}^{2}\right) u_{2}=0 & \text { in } \Omega_{2}:=\mathbf{R}^{2} \backslash \bar{\Omega}_{1} & \mu \frac{\partial u_{1}}{\partial n}=\frac{\partial u_{2}}{\partial n}+\psi_{0}
\end{array}\right\} \quad \text { on } \Gamma
$$

subject to the Sommerfeld radiation condition

$$
\frac{\partial u_{2}}{\partial R}-i k_{2} u_{2}=o\left(R^{-1 / 2}\right), \quad u_{2}=O\left(R^{-1 / 2}\right) \quad \text { as }|x|=R \rightarrow \infty .
$$

In the case of scattering problems, $u_{1}$ and $u_{2}$ denote the refracted and scattered field, respectively, and $v_{0}$ and $\psi_{0}$ are the boundary trace and the normal derivative of the incident field $u_{0}$. In [4] the above transmission problem is reduced to a system of boundary integral equations on $\Gamma=\partial \Omega_{1}$ for the Cauchy data $v_{1}=u_{1}\left|\Gamma, \psi_{1}=\left(\partial u_{1} / \partial n\right)\right|_{\Gamma}$ :

$$
\begin{aligned}
H\left(\begin{array}{c}
v_{1} \\
\psi_{1}
\end{array}\right) & :=\frac{1}{2}\left(\begin{array}{cc}
-\left(K_{1}+K_{2}\right) & V_{1}+\mu V_{1} \\
D_{1}+(1 / \mu) D_{2} & K_{1}^{\prime}+K_{2}^{\prime}
\end{array}\right)\left(\begin{array}{c}
v_{1} \\
\psi_{1}
\end{array}\right) \\
& =\left(\begin{array}{c}
v_{0} \\
(1 / \mu) \psi_{0}
\end{array}\right)
\end{aligned}
$$

where $(j=1$ or 2$)$

$$
\begin{gathered}
V_{j} \psi(z)=-2 \int_{\Gamma} \psi(\zeta) \gamma_{j}(z, \zeta) d s_{\zeta} \\
K_{j} v(z)=-2 \int_{\Gamma} v(\zeta) \frac{\partial}{\partial n_{\zeta}} \gamma_{j}(z, \zeta) d s_{\zeta} \\
z \in \Omega_{j}, \quad D_{j} v_{j}=-\left.\frac{\partial}{\partial n} K_{j} v_{j}\right|_{\Gamma}
\end{gathered}
$$


and $K_{j}^{\prime}$ is the adjoint operator of $K_{j}$.

$$
\begin{aligned}
\gamma_{j}(z, \zeta) & =-\frac{i}{4} H_{0}^{(1)}\left(k_{j}|z-\zeta|\right) \\
& =\frac{1}{2 \pi} \log |z-\zeta|+O\left(|z-\zeta|^{-1}\right)
\end{aligned}
$$

is the fundamental solution of the Helmholtz equation $\Delta w=-k_{j}^{2} w$ in $\Omega_{j}$ where $H_{0}^{(1)}$ is the Hankel function of first order and degree zero.

It is shown in [4] that the operator

$$
H: H^{1 / 2}(\Gamma) \times H^{-1 / 2}(\Gamma) \longrightarrow H^{1 / 2}(\Gamma) \times H^{-1 / 2}(\Gamma)
$$

is bijective if and only if the homogeneous transmission problem (60) as well as the adjoint problem, obtained by interchanging $\Omega_{1}$ and $\Omega_{2}$, have only the trivial solution. This is assumed in the following. From the regularity results in [4] follows that, for piecewise analytic data $v_{0}$ and $\psi_{0}$, the solution $\left(v_{1}, \psi_{1}\right)$ of (61) has expansions of the form (9), (10) with $\alpha_{k_{j}}=k \alpha_{j}$ and $\alpha_{j}$ being a zero of the transcendental equation

$$
\frac{\sin \left(\pi-\omega_{j}\right) \alpha}{\sinh \pi \alpha}= \pm\left(\frac{\mu+1}{\mu-1}\right)
$$

The boundary element Galerkin scheme for (61) reads (for the definition of $\langle\cdot, \cdot\rangle$ see [4]): Find $\left(v_{N}, \psi_{M}\right) \in Y_{N} \times X_{M}$ such that

$$
\begin{aligned}
&\left\langle H\left(\begin{array}{c}
v_{N} \\
\psi_{M}
\end{array}\right),\right.\left.\left(\begin{array}{c}
w \\
\phi
\end{array}\right)\right\rangle \\
& \forall(w, \phi)=\left\langle\left(\begin{array}{c}
v_{0} \\
(1 / \mu) \psi_{0}
\end{array}\right),\left(\begin{array}{l}
w \\
\phi
\end{array}\right)\right\rangle \\
& Y_{N} \times X_{M}
\end{aligned}
$$

where $Y_{N}, X_{M}$ are finite dimensional subspaces of $H^{1 / 2}(\Gamma)$ and $H^{-1 / 2}(\Gamma)$ with $\operatorname{dim} Y_{N}=N$ and $\operatorname{dim} X_{M}=M$. Since the operator $H$ satisfies a Gårding's inequality in $H^{1 / 2}(\Gamma) \times H^{-1 / 2}(\Gamma)$ this boundary element Galerkin scheme converges quasioptimally in the energy norm, i.e.,

$$
\begin{aligned}
& \left\|v_{N}-v_{1}\right\|_{H^{1 / 2}(\Gamma)}+\left\|\psi_{M}-\psi_{1}\right\|_{H^{-1 / 2}(\Gamma)} \\
& \quad \leq \inf _{(w, \phi) \in Y_{N} \times X_{M}}\left\{\left\|v_{1}-w\right\|_{H^{1 / 2}(\Gamma)}+\left\|\psi_{1}-\phi\right\|_{H^{-1 / 2}(\Gamma)}\right\} .
\end{aligned}
$$


Next we choose $X_{N}=S^{P-1,0}\left(\Gamma_{\sigma}^{n}\right)$ and $Y_{N}=S^{P, 1}\left(\Gamma_{\sigma}^{n}\right)$ as in Section 4 and obtain the exponential convergence of the hp-version of the Galerkin scheme (63) for the transmission problem (60).

Proposition 1. Let $v_{0}$ and $\psi_{0}$ in (60) be piecewise analytic such that there holds one of the assumptions of Corollaries 1-3. Then, for the error between the Galerkin solution $v_{N} \in S^{P, 1}\left(\Gamma_{\sigma}^{n}\right), \psi_{N} \in S^{P-1,0}\left(\Gamma_{\sigma}^{n}\right)$ and the exact solution of (61), there holds

$$
\left\|v_{1}-v_{N}\right\|_{H^{1 / 2}(\Gamma)}+\left\|\psi_{1}-\psi_{N}\right\|_{H^{-1 / 2}(\Gamma)} \leq C e^{-b \sqrt{N}}
$$

if the degrees $P$ are suitably chosen. Here $N=\operatorname{dim} S^{P, 1}\left(\Gamma_{\sigma}^{n}\right)=$ $\operatorname{dim} S^{P-1,0}\left(\Gamma_{\sigma}^{n}\right)$ and $C$ and $b$ are constants depending on $\sigma$ but not on $N$.

Proof. Firstly, we observe that for piecewise analytic data $v_{0}, \psi_{0}$ fulfilling one of the assumptions of Corollaries 1-3, the solution $\left(v_{1}, \psi_{1}\right)$ of $(61)$ belongs to $B_{\beta}^{2}(\Gamma) \times B_{\beta}^{1}(\Gamma)$ with $1>\beta>3 / 2-\alpha_{\min }$ where $\alpha_{\min }$ is the smallest zero of (62). Therefore, application of the analysis in Section 4 yields the assertion of the proposition.

Remark 5. For the transmission problem (60) with $k_{1}=k_{2}=0$ the exponential convergence of the hp-version of the BEM has been shown recently in $[8]$.

Remark 6. Two-dimensional crack problems in linear elasticity can be converted into first kind integral equations (see $[\mathbf{1 0}, \mathbf{1 3}])$. For example, let us consider the Neumann crack problem for the domain $\Omega_{\Gamma}$ exterior to an arc $\Gamma$ : Find $u \in H_{\mathrm{loc}}^{1}\left(\Omega_{\Gamma}\right)$ such that $\Delta^{*} u \equiv$ $\mu \Delta u+(\lambda+\mu) \operatorname{grad} \operatorname{div} u=0$ in $\Omega_{\Gamma}=\mathbf{R}^{2} \backslash \bar{\Gamma}$ and

$$
\left.T(u)\right|_{\Gamma_{1}}=\psi_{1},\left.\quad T(u)\right|_{\Gamma_{2}}=\psi_{2}
$$

for given $\psi_{i} \in H^{-1 / 2}(\Gamma), i=1,2$, where $T$ denotes the traction operator on the sides $\Gamma_{1}$ and $\Gamma_{2}$ of $\Gamma$ and $\lambda, \mu$ are given Lamé constants. Under appropriate conditions, e.g., assuming a decaying condition for $u$ at infinity, this problem can be converted into the integral equation

$$
D \phi(x)=-T_{x} \int_{\Gamma}\left(T_{y}(E(x, y))\right)^{T} \phi(y) d s_{y}=f(x), \quad x \in \Gamma
$$




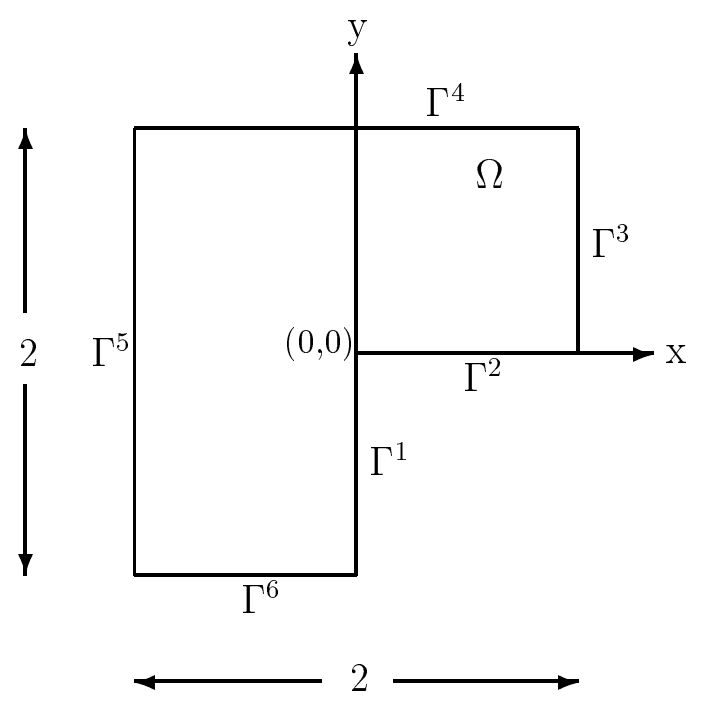

FIGURE 1. $L$-shaped domain.

for the jump $\phi \equiv[u]=\left.u\right|_{\Gamma_{1}}-\left.u\right|_{\Gamma_{2}}$ with the fundamental solution of the Navier operator $\Delta^{*}$

$$
E(x, y)=\frac{\lambda+3 \mu}{4 \pi \mu(\lambda+2 \mu)}\left\{\log \frac{1}{|x-y|} I+\frac{\lambda+\mu}{\lambda+3 \mu} \frac{(x-y)(x-y)^{T}}{|x-y|^{2}}\right\} .
$$

Here $T$ denotes the transposed tensor, $I$ is the identity matrix and $f$ is given via $\psi_{1}$ and $\psi_{2}$ (see [13]). It is shown in [13] that the solution $\phi$ of the hypersingular integral equation (64) behaves like $x^{1 / 2}\left(d_{1}+d_{2} x+d_{3} x^{2}+\cdots\right), d_{j} \in \mathbf{R}$, near the crack tip $x=0$, i.e., like $v$ in (10) with $\omega=2 \pi$ and $\alpha_{k}=1 / 2+k, k \geq 0$ integer. Therefore $\phi \in B_{\beta}^{1}(\Gamma)$ for $0<\beta<1$, cf. Lemma 3 .

The operator $D$ in (64) satisfies a Gårding's inequality in $\tilde{H}^{1 / 2}(\Gamma)$ (see [13]) and therefore the corresponding Galerkin scheme converges quasioptimally in $\tilde{H}^{1 / 2}(\Gamma)$. Therefore the analysis in Section 4 applies also to the integral equation (64) yielding exponentially fast convergence for the Galerkin solution of the hp-version with geometric meshes in the $L^{2}$-norm. This result is also true of the energy norm $\|\cdot\|_{\tilde{H}^{1 / 2}(\Gamma)}$ as can be seen by a more detailed analysis, cf. [9]. 




Geometric mesh $\Gamma_{0.5}^{0}$

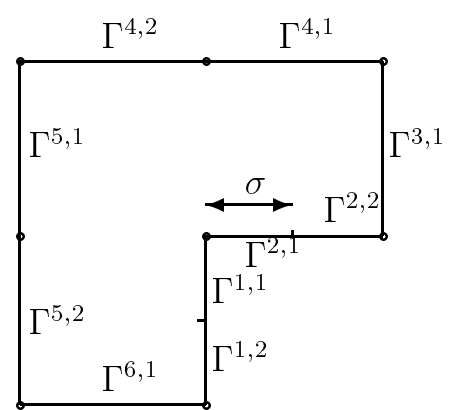

Geometric mesh $\Gamma_{0.5}^{1}$



Geometric mesh $\Gamma_{0.5}^{2}$

FIGURE 2. Geometric meshes $\Gamma_{\sigma}^{n}, 0 \leq n \leq 2, \sigma=0.5$.

6. Numerical results. Now we present numerical results for the Dirichlet problem using the h-, p- and hp-versions of the Galerkin method. In our example we use for $\Omega$ the $L$-shaped domain shown in Figure 1 and choose in (1) $f:=\left.u\right|_{\Gamma}$ for $u(z):=\operatorname{Im}\left(z^{2 / 3}\right)$ with $z=x+i y$. Then, due to Lemma $3, u$ belongs to $B_{\beta}^{2}(\Gamma), 5 / 6<\beta<1$, with the occurrence of a singularity at the origin. Thus, for the solution $\psi=\left.(\partial u / \partial n)\right|_{\Gamma}$ of $(3)$ there holds $\psi \in B_{\beta}^{1}(\Gamma), 5 / 6<\beta<1$.

Theorem 2 proves the exponentially fast convergence of the hp-version with geometrically refined meshes. Three samples in the sequence of geometric meshes for $\sigma=0.5$ are shown in Figure 2. Note that, due to 
the only singularity at the origin, the meshes are just refined towards the reentrant corner.

For the implementation of the linear system,

$$
\left\langle V \psi_{N}, \phi\right\rangle_{L^{2}(\Gamma)}=\langle(1+K) f, \phi\rangle_{L^{2}(\Gamma)}, \quad \forall \phi \in X_{N},
$$

we rewrite the righthand side as

$$
\langle(1+K) f, \phi\rangle_{L^{2}(\Gamma)}=\left\langle f,\left(1+K^{\prime}\right) \phi\right\rangle_{L^{2}(\Gamma)}
$$

where $K^{\prime}$ is the adjoint of the double layer potential. Then we use analytical formulas for the inner integrations and calculate the outer integrals by a Gaussian quadrature formula. To compute the error in the energy norm of the Galerkin solution $\psi_{N}$, we apply the relation

$$
\begin{aligned}
\left\|\psi-\psi_{N}\right\|_{H^{-1 / 2}(\Gamma)}^{2} & \simeq\left\|\psi-\psi_{N}\right\|_{V}^{2} \\
& :=\left\langle V\left(\psi-\psi_{N}\right), \psi-\psi_{N}\right\rangle_{L^{2}(\Gamma)} \\
& =\langle V \psi, \psi\rangle_{L^{2}(\Gamma)}-\left\langle V \psi_{N}, \psi_{N}\right\rangle_{L^{2}(\Gamma)} \\
& =\|\psi\|_{V}^{2}-\left\|\psi_{N}\right\|_{V}^{2}
\end{aligned}
$$

For $\|\psi\|_{V}$ we take an approximation obtained by extrapolating the norms $\left\|\psi_{h}\right\|_{V}$ of Galerkin solutions $\psi_{h}$ of the $h$-version. For more details, we refer to $[\mathbf{6}]$.

Figure 3 presents the relative errors in the energy norm for the two sequences of geometric meshes $\sigma=0.5$ and $\sigma=0.15$. For comparison, we also show the numerical results for the h- and p-versions with quasiuniform meshes. These versions converge algebraically with rates of $2 / 3$ and $4 / 3$, respectively (cf. [11] and [6]). The convergence of the hp-version with geometric meshes is better than algebraic as confirmed by the downwardly curved lines whereas the curves for the h- and the pversion are approximately straight lines. The figure also demonstrates the influence of the mesh-parameter $\sigma$ which gives a rapidly convergent method for $\sigma \approx 0.15$. After giving a relative accuracy of about $0.2 \%$ the error blows up again. However, we note that the condition number increases exponentially in the number of unknowns if the hp-version with geometric meshes is performed. Therefore, since we are using quadrature formulas, the upward kick in the curve is not surprising.

Acknowledgment. We gratefully acknowledge suggestions made by the reviewers. 


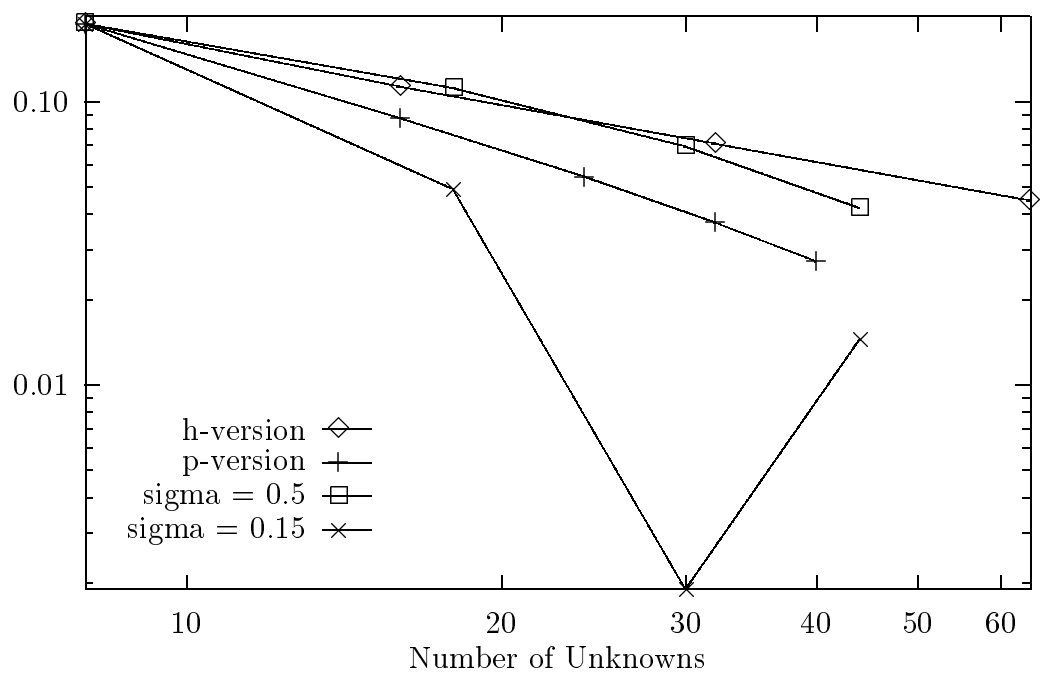

FIGURE 3. The relative errors in the energy norm for the h-, p- and hpversions.

\section{REFERENCES}

1. I. Babuška, B.Q. Guo and E.P. Stephan, On the exponential convergence of the $h-p$ version for boundary element Galerkin methods on polygons, Math. Methods Appl. Sci. 12 (1990), 413-427.

2. M. Costabel, Boundary integral operators on Lipschitz domains: Elementary results, SIAM J. Math. Anal. 19 (1988), 613-626.

3. M. Costabel and E.P. Stephan, Boundary integral equations for mixed boundary value problems in polygonal domains and Galerkin approximations, in Mathematical models and methods in mechanics (W. Fiszdon and K. Wilmanski, eds.), Banach Centre Publ., Warsaw, 1985.

4. - A direct boundary integral equation method for transmission problems, J. Math. Anal. Appl. 106 (1985), 367-413.

5. M. Costabel, E.P. Stephan and W.L. Wendland, On boundary integral equations of the first kind for the bi-Laplacian in a polygonal domain, Ann. Scuola Norm. Sup. Pisa, Cl. Ser (4) 10 (1983), 197-241.

6. V.J. Ervin, N. Heuer and E.P. Stephan, On the $h-p$ version of the boundary element method for Symm's integral equation on polygons, Comput. Methods Appl. Mech. Engrg. 110 (1993), 25-38. 
7. B.Q. Guo and I. Babuška, The h-p version of the finite element method, Part 1: The basic approximation results, Comp. Mech. 1 (1986), 21-41.

8. B.Q. Guo, N. Heuer and E.P. Stephan, The h-p version of the boundary element method for transmission problems with piecewise analytic data, SIAM J. Numer. Anal. 33 (2) (1996), 789-808.

9. N. Heuer, M. Maischak and E.P. Stephan, The hp-version of the boundary element method for screen problems, Institut für Angewandte Mathematik, Hanover, Germany, 1995, preprint.

10. G.C. Hsiao, E.P. Stephan and W.L. Wendland, On the Dirichlet problem in elasticity for a domain exterior to an arc, J. Comput. Appl. Math. 34 (1991), 1-19.

11. E.P. Stephan and M. Suri, The $h-p$ version of the boundary element method on polygonal domains with quasiuniform meshes, Math. Modeling Numer. Anal. 25 (1991), 783-807.

12. E.P. Stephan and W.L. Wendland, Remarks to Galerkin and least squares methods with finite elements for general elliptic problems, Manuscripta Geodaetica 1 (1976), 93-123.

13. W.L. Wendland and E.P. Stephan, A hypersingular boundary integral method for two-dimensional screen and crack problems, Arch. Rational Mech. Anal. 112 (1990), 363-390.

LANDESREChENZENTRUM FÜr DEN WisSENSChaftSBEREICH, C/O Universität Bremen, Germany

Institut für Angewandte Mathematik, Universität Hannover, Germany 